\title{
FIBRINOLYTIC ACTIVITY OF HEMOLYTIC STREPTOCOCCI. THE DEVELOPMENT OF RESISTANCE TO FIBRINOLY- SIS FOLLOWING ACUTE HEMOLYTIC STREPTO- COCCUS INFECTIONS
}

\author{
By WILLIAM S. TILLETT, L. B. EDWARDS, AND R. L. GARNER \\ (From the Biological Division of the Department of Medicine of the Johns Hopkins \\ Medical School and Hospital, Baltimore)
}

(Received for publication September 6, 1933)

In a recently published report (1) results were presented which demonstrate that broth cultures of hemolytic streptococci are capable of rapidly liquefying the fibrin-clot of normal human plasma. A description was given of the presence of fibrinolytic substances in cultures, as well as in sterile filtrates, and of the conditions of fibrin coagulation which influence the occurrence of liquefaction. The fibrinolysin was found, by the experimental method described, in every strain of hemolytic streptococcus isolated from patients. On the contrary, cultures of other species of bacteria, also derived from human beings, were, in comparable tests, unable to cause fibrinolysis.

This special characteristic of human strains of hemolytic streptococci is being investigated both with reference to its biochemical properties and also for the purpose of determining whether or not the fibrinolytic activity of the living organisms might be a factor in hemolytic streptococcus infections. The results of the chemical investigations will be subsequently published.

The purpose of this article is to describe the resistance to streptococcal fibrinolysis which develops in the blood of patients following acute infections with hemolytic streptococci, and to contrast the insusceptibility exhibited by the blood of these patients with the results of similar tests on the plasma of patients suffering from other bacterial infections.

In addition to the observations which have been made with the blood from patients, tests have been performed with the fibrin-clot of plasma from healthy normal adults.

A fourth group of cases, whose plasma has been similarly tested, consists of patients in the dispensary or hospital who either presented no abnormal physical findings at the time blood was withdrawn, or in whom the low grade chronic ailment was deemed least likely to influence the fibrinolytic test with which this report is concerned. This group, although not normal, has been utilized in an attempt to obtain tests with blood from individuals in different age groups, normal healthy representatives of which 
were not available. For the purpose of this article they are tentatively considered as normal.

A test consists in mixing oxalated plasma with living, full grown, broth cultures of hemolytic streptococci, and then inducing clot formation by the addition of $\mathrm{CaCl}_{2}$. With the fibrin-clot from the plasma of certain individuals, liquefaction is completed in a few minutes. On the other hand, when the plasma of other patients is coagulated in the presence of active cultures, the resultant coagulum resists liquefaction completely or delays the process. The interval of time, therefore, between clot formation and clot dissolution is used as an index of susceptibility or resistance to the fibrinolytic principle of active cultures.

No attempt has been made to establish arbitrarily a rate of dissolution to serve as a sharp dividing line between normal and abnormal. Minor variations in the quantity of fibrin in a given sample of clotted plasma or in the amount of fibrinolysin in the cultures may change the time required for dissolution by several minutes. However, by using constant amounts of plasma and culture in all the tests, the limits of experimental error due to the uncontrolled quantitative variation just referred to, have been minimized to such a degree that they do not cause a variation in dissolution time of more than 10 to 15 minutes. When, therefore, the difference in the time of liquefaction between the fibrin-clot of a patient's blood and that of a known susceptible is proved to be a matter of several hours, the delayed rate has been considered significant. As an aid in interpreting the importance of the time element in fibrinolytic tests, the following classification has seemed practicable.

Dissolution in less than 30 minutes-Highly susceptible.

Dissolution in 30 to 60 minutes-Susceptible.

Dissolution in 1 to 8 hours-Definite resistance.

Dissolution in 8 to 24 hours-Marked resistance.

No dissolution in 24 hours-Maximum resistance.

All experiments were arbitrarily terminated at the end of 24 hours in water bath at $37.5^{\circ} \mathrm{C}$.

\section{MATERIALS AND METHODS}

For a more complete description of all the details, the reader is referred to the previous article (1). As formerly stated, the experimental conditions which best promote the occurrence of rapid fibrinolysis consist in mixing cultures with oxalated plasma before inducing clot formation.

Cultures. A strain of hemolytic streptococcus, designated Co., and known to be highly active in the production of fibrinolytic substances, has been employed in most of the tests. Fresh 18 to 24 hour cultures were utilized. Plain meat infusion broth, containing 0.05 per cent dextrose, was the most satisfactory medium. Occasionally broth cultures, kept in the icebox, were used for 2 to 4 days. When, by testing the old cultures with susceptible fibrin-clot, the rate of dissolution was delayed, a fresh culture was employed. 
In several instances, the hemolytic streptococci isolated from a patient, were used in tests with the patients' plasma. In such observations, however, no specificity was demonstrable, and no special advantage was obtained by employing strains and plasma from the same patient.

Although sterile filtrates have been found to contain the fibrinolytic principle in large quantities, whole broth cultures were employed in all the tests reported in this communication. Since the tests were designed to determine resistance to clot liquefaction, the more potent cultures have been used instead of filtrate, in order to make the test more stringent and exacting.

Anticoagulant. Potassium oxalate has been regularly employed in amounts of 0.02 gram of oxalate to 10 cc. of blood. A 2 per cent solution is made in distilled water. One cubic centimeter of this solution is placed in small bottles which are heated in a dry air sterilizer until all water has evaporated. Ten cubic centimeters of blood, immediately after withdrawal, are mixed with the dried powder.

Coagulant. $0.25 \mathrm{cc}$. of a 0.25 per cent solution of $\mathrm{CaCl}_{2}$ in 0.85 per cent salt solution consistently clotted $0.2 \mathrm{cc}$. of oxalated plasma in 8 to 20 minutes. The solution of calcium chloride was sterilized by immersion in boiling water for $\mathbf{3 0}$ minutes.

In the experiments reported in this article samples of plasma were always tested within 24 hours of the time blood was withdrawn. This fact is important since coagulation with $\mathrm{CaCl}_{2}$ is delayed or inhibited in oxalated plasma which is several days old.

Description of test. The following quantities of the various ingredients, which have been uniformly employed, constitute a "standard" test: 0.2 cc. of oxalated plasma is diluted with $0.8 \mathrm{cc}$. of physiological salt solution. To this 1 to 5 dilution of plasma, $0.5 \mathrm{cc}$. of broth culture of test organisms is added and well mixed. 0.25 cc. of 0.25 per cent solution of $\mathrm{CaCl}_{2}$ is then added and well mixed. The tubes are immediately placed in water bath at $37.5^{\circ} \mathrm{C}$. Solid coagulation usually occurs in 6 to 20 minutes; the average time is about 10 minutes. Solid coagulation is considered to be effected when the tubes can be inverted without affecting the solid form of clot which adheres to the bottom and sides of the tube; usually no fluid, or only a small drop, escapes from the solid clot on inversion of the tube.

The tubes are allowed to remain in the water bath under continual observation. Dissolution of the clot is recorded as being complete when all evidence of solid fibrin has disappeared, and the contents of the tube are completely fluid.

By recapitulation, a test is as follows :

$0.2 \mathrm{cc}$. oxalated plasma $+0.8 \mathrm{cc}$. physiological salt solution $+0.5 \mathrm{cc}$. culture +0.25 cc. $\mathrm{CaCl}_{2}$.

All tests in which the plasma-clot was resistant to dissolution were arbitrarily terminated after 24 hours incubation.

Before proceeding with a description of the results of fibrinolytic tests obtained with the blood of patients, some idea of the "normal" rate of reaction may be derived from the tests performed with the fibrin-clot from the plasma of healthy adults and of the specially selected group of dispensary and hospital cases previously mentioned.

Thirty healthy adults were chosen from students, technicians, and members of the hospital staff. In this group it was possible to select those who were healthy at the time the test was made, and who gave no history 
of acute infections during the past winter. The results are recorded in Table I.

TABLE I

Fibrinolytic tests with plasma-clot from the blood of normal, healthy adults

\begin{tabular}{|c|c|c|c|c|c|c|c|c|c|}
\hline \multirow[t]{2}{*}{ Individual } & \multirow[t]{2}{*}{$\begin{array}{l}\text { Age } \\
\text { dec- } \\
\text { ade }\end{array}$} & \multirow[t]{2}{*}{ Sex } & \multicolumn{2}{|c|}{$\begin{array}{l}\text { Time required for } \\
\text { complete dissolution } \\
\text { of plasma-clot* }\end{array}$} & \multirow{3}{*}{$\begin{array}{c}\text { Individual } \\
16\end{array}$} & \multirow{3}{*}{$\begin{array}{l}\begin{array}{c}\text { Age } \\
\text { dec- } \\
\text { ade }\end{array} \\
\text { 3rd }\end{array}$} & \multirow{3}{*}{$\begin{array}{c}\text { Sex } \\
F\end{array}$} & \multicolumn{2}{|c|}{$\begin{array}{l}\text { Time required for } \\
\text { complete dissolution } \\
\text { of plasma-clot* }\end{array}$} \\
\hline & & & hours & minutes & & & & hours & minutes \\
\hline 1 & 3rd & $\mathbf{M}$ & & 15 & & & & & 10 \\
\hline 2 & $3 r d$ & $\mathbf{M}$ & & 8 & 17 & 3rd & $\mathbf{M}$ & & 16 \\
\hline 3 & 3rd & $\mathbf{M}$ & & 12 & 18 & $3 r d$ & $\mathbf{M}$ & & 10 \\
\hline 4 & 3rd & $\mathbf{M}$ & & 15 & 19 & 3rd & $\mathbf{M}$ & & 20 \\
\hline 5 & $3 r d$ & $\mathbf{M}$ & & 25 & 20 & 3rd & $\mathbf{M}$ & & 5 \\
\hline 6 & 3rd & $\mathrm{F}$ & 1 & 5 & 21 & $3 r d$ & $\mathbf{M}$ & & 45 \\
\hline 7 & $3 r d$ & $\mathrm{~F}$ & & 5 & 22 & $3 r d$ & $\mathbf{M}$ & 1 & \\
\hline 8 & $3 r d$ & $\mathbf{M}$ & & 40 & 23 & 4 th & $\mathbf{M}$ & & 15 \\
\hline 9 & $3 r d$ & F & 1 & 35 & 24 & 4th & $\mathbf{M}$ & & 10 \\
\hline 10 & $3 r d$ & $\mathbf{M}$ & & 15 & 25 & 4 th & $\mathbf{M}$ & 2 & 20 \\
\hline 11 & 3rd & $\mathbf{M}$ & & 10 & 26 & 4th & $\mathbf{M}$ & & 15 \\
\hline 12 & $3 r d$ & $\mathbf{M}$ & 4 & & 27 & 4 th & $\mathrm{F}$ & & 25 \\
\hline 13 & $3 r d$ & $\mathbf{M}$ & 3 & & 28 & 4 th & $\mathbf{F}$ & & 10 \\
\hline 14 & 3rd & $\mathbf{M}$ & 1 & 15 & 29 & 4th & $\mathbf{M}$ & & 25 \\
\hline 15 & 3rd & $\mathbf{M}$ & 3 & & 30 & 4th & $\mathbf{M}$ & & 10 \\
\hline
\end{tabular}

* Repeated tests have been made with many of the specimens of blood. The average rate is recorded in the table.

The normal controls listed above were all in the 3rd and 4th decades of life. There are 24 males and 6 females. In this small series the plasmaclot of 70 per cent was liquefied in less than one hour; only 2 of these required longer than 30 minutes. The time of clot dissolution in the remaining 30 per cent ranged from 1 hour and 5 minutes to 4 hours. Variation in the rate of fibrinolysis exhibited by the blood of different healthy individuals cannot, as yet, be interpreted. Consideration of this point will be given later in this article when the results of repeated tests are described.

Table II contains the results of tests performed with the blood of 30 patients who were selected with special reference to age. The nature of their chronic illnesses is recorded in the table, and, where possible, a history of recent acute infections is noted. Fourteen of the patients are under 13 years of age, two are in the 5 th decade of life, and the ages of the remaining fourteen range from 50 to 73 years.

The children included in Table II came to the Dispensary of the Harriet Lane Home for Children. Eight of these were receiving routine antiluetic treatment for congenital syphilis. Plasma was obtained from the others at the time blood was taken for the Wassermann reaction. The plasma-clot from seven of the children liquefied in less than 1 hour, and the dissolution time in the remaining seven ranged from 2 hours and 15 


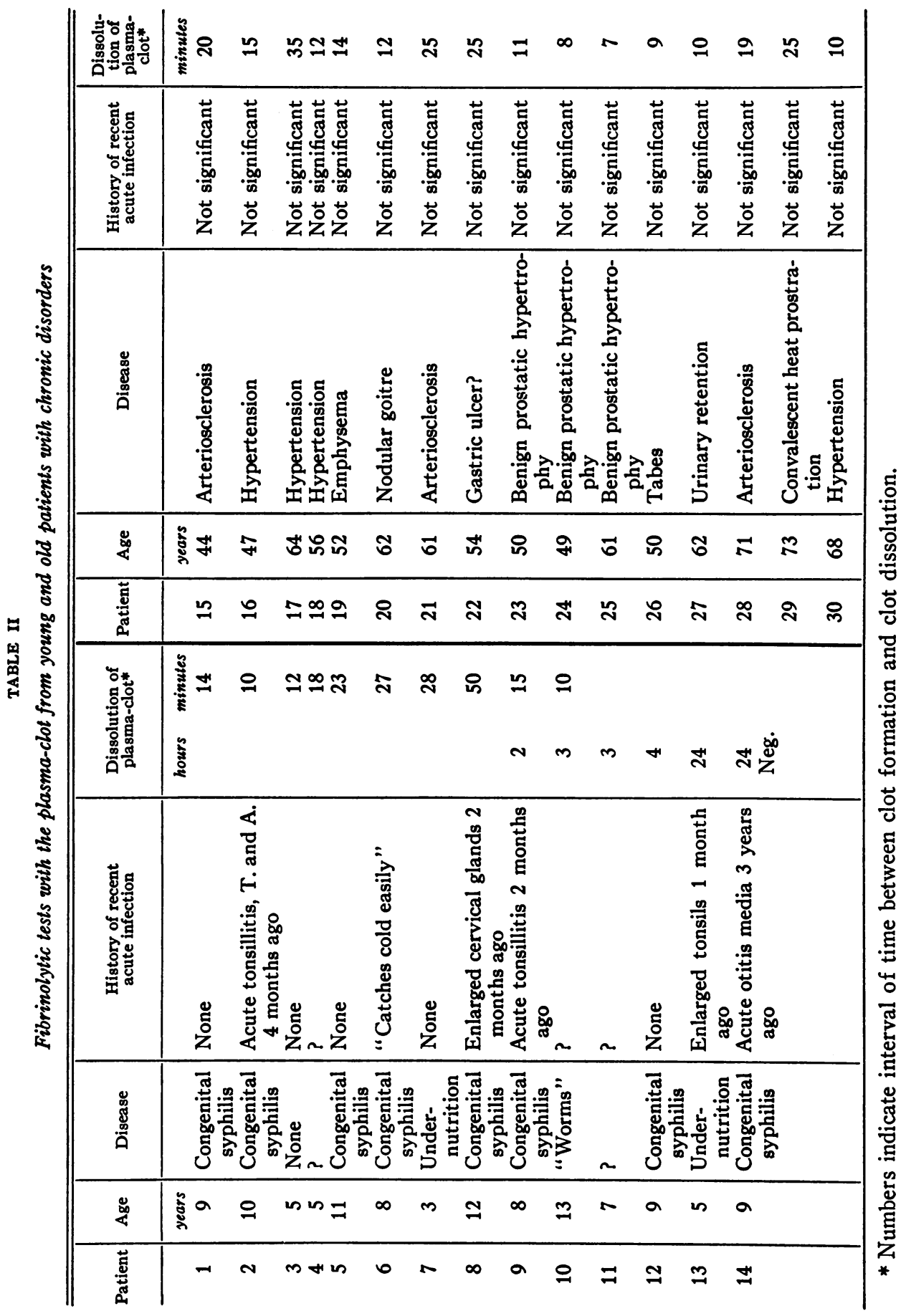


minutes to 24 hours. All of these patients were in good general health at the time blood was obtained. Although the number of cases is too few to justify conclusions, the frequency of acute upper respiratory infections in children may account for the relatively high proportion of cases with increased resistance.

With the plasma-clot from the blood of patients in the older group, fibrinolytic rate was rapid in each instance. Whether, in older, mildly debilitated individuals, uniform susceptibility is significant, cannot be stated.

By combining the tests, which are recorded in Tables I and II, the group of "normal" persons consists of sixty individuals. The time of clot dissolution with the blood in 75 per cent of these tests is classified as susceptible; 68.3 per cent are highly susceptible. These results are not presented for the purpose of attempting to fix arbitrarily an absolute normal but they serve to disclose the relative rate of streptococcal fibrinolysis among a group of individuals not suffering from acute infection.

Repeated fibrinolytic tests with plasma-clot of blood from patients, before and after recovery from hemolytic streptococcus infections

Twelve patients suffering from various manifestations of acute infection with hemolytic streptococci were used for these observations. In each instance the clinical diagnosis was definite, and hemolytic streptococci were isolated from the patients.

In each case specimens of blood were obtained during the acute stages of disease as well as during convalescence. It has also been possible to follow most of the cases for one to five months after discharge from the hospital. The opportunity, therefore, of repeatedly procuring samples of plasma has served as a means of correlating the clinical course of the infection with changes in the rate of dissolution of the plasma-clot from these patients, and of determining, in addition, the length of time, after recovery, that anti-fibrinolytic principles remain in the blood.

A brief account of the clinical course of each case is given. Individual charts are presented which diagrammatically record the course of the temperature, and the more important signs and symptoms. The time required for dissolution of the plasma-clot from the patients' blood is compared to a test, simultaneously done, with the plasma-clot of a normal blood known to be susceptible.

The cases, which are given in detail with accompanying charts, consist of :

1. Four cases of erysipelas. Three recovered and one died.

2. Three cases of scarlet fever. All recovered; one developed purulent sinusitis.

3. Five cases of acute tonsillitis. All recovered. In two cases convalescence was uneventful; in three others, acute purulent complications occurred. 
Patient Br. (See Chart 1). History Number 47,893. White, male, age 38 years. Admitted: February 17, 1933. Disease : Erysipelas of face.

Résumé of clinical course. The patient was admitted on the 3d day of disease. Two days before entry he had become weak, dizzy, alternately chilly and feverish, and noticed that his eyes were swollen. He remained acutely ill and the swelling spread to his forehead and nose.

On admission the temperature was $106.4^{\circ}$. There was a typical erysipelatous lesion involving forehead, eyelids, nose, which spread in butterfly distribution

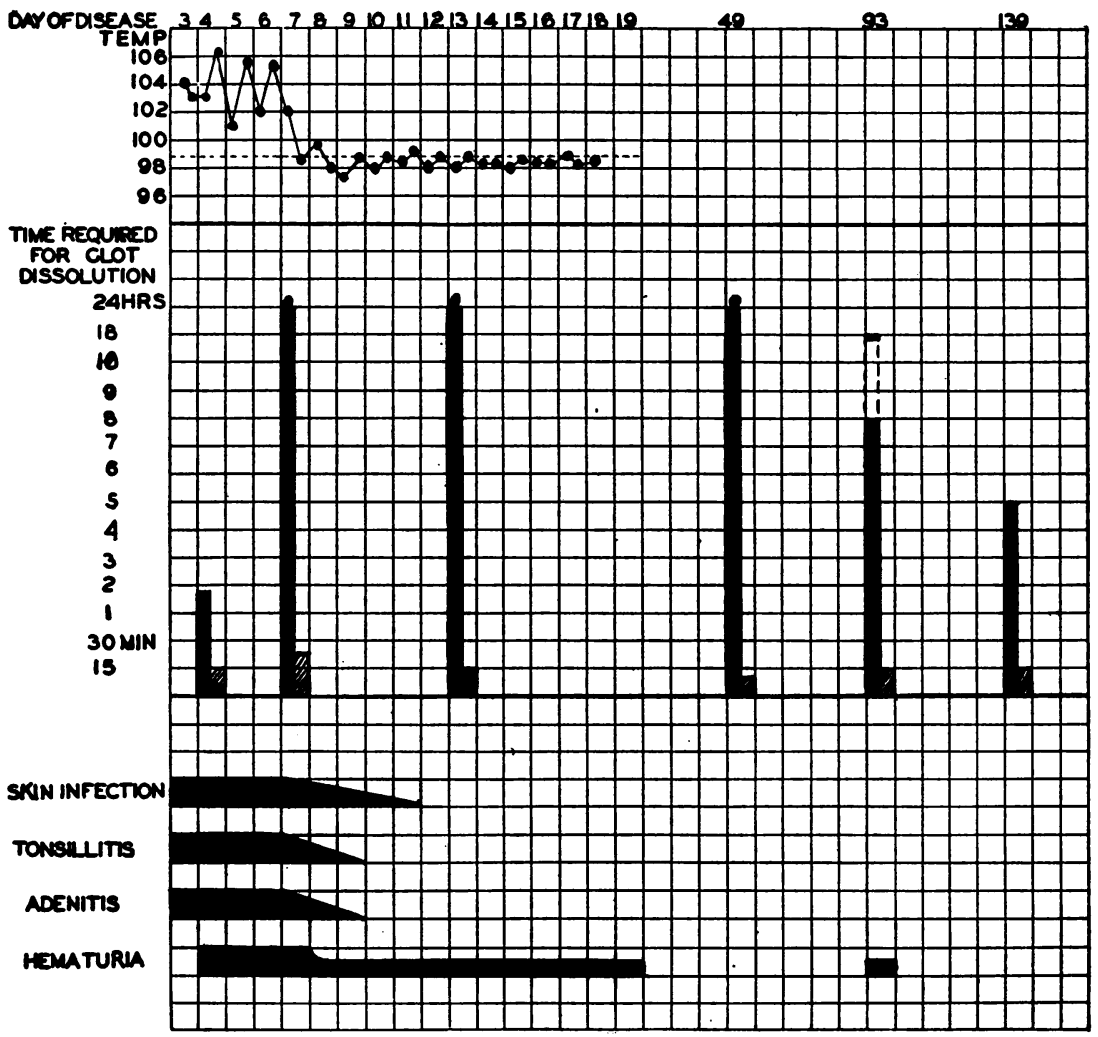

Chart 1. Patient Br., Male, Age 38 Years. Disease, Erysipelas

In this and subsequent charts, the upright columns which are recorded at intervals represent fibrinolytic tests with "standard" amounts of plasma and cultures of hemolytic streptococci.

The day on which blood was taken is indicated by the "Day of Disease" at the top of the chart which corresponds to the columns.

The solid black column represents a test with the patient's blood. A flat top to the column indicates the time at which liquefaction was complete; a round dot at the top of the column signifies that the experiment was terminated even though dissolution had not occurred; the section with interrupted lines, which continue above a black column, represents a period of incomplete observation.

The diagonally striated column indicates a control test, simultaneously performed with plasma from a known susceptible. 
over cheeks. The tonsils were acutely inflamed, and the cervical glands were enlarged and tender.

Laboratory findings. White blood cells, 13,800 per c.mm. Throat culture: Many colonies of hemolytic streptococci. Blood culture: Sterile. Urine: Trace of albumin, many red blood cells.

Temperature remained high and swinging for four days, during which time the erysipelas spread over the entire forehead and face and involved the lobes of the ears. On the eighth day of disease the temperature fell rather abruptly to normal, the lesion ceased to spread, and symptoms subsided. Recovery was uneventful except for the presence of a few red blood cells in his urine which were present at the time of discharge from the hospital.

Fibrinolytic tests. Samples of blood were obtained on the 3rd day before recovery (4th day of disease), on the day of abrupt drop in temperature (7th day of disease), on the 6th day after recovery (13th day of disease), and on 3 different occasions after discharge from the hospital, the last of which was about four and one-half months after recovery.

The fibrin-clot from the plasma obtained during the stage of acute illness was liquefied by active cultures of hemolytic streptococci at a somewhat slower rate than the normal control. The plasma clot from other cases taken during the acute stage of disease has frequently showed a slightly delayed dissolution time. Consideration of this point will be subsequently given. The observation of special interest made with the blood from this case of erysipelas is the sudden appearance on the day of recovery of complete insusceptibility to fibrinolysis. The plasma taken on that day, when clotted in the presence of active culture, remained solid during the 24 hour test period. The potency of the test culture was proved by the rapid dissolution of normal plasma-clot. The resistance to fibrinolysis remained maximum in two subsequent tests on the 7th and 42nd days in convalescence. When tested again about 3 months later, resistance was still high-requiring 8 hours for dissolution-although a decrease from maximum had occurred. In the last test, four and one-half months after recovery, fibrinolysis required 5 hours, indicating a slowly progressive diminution in resistance.

Comment. In this case of erysipelas, maximum resistance to fibrinolysis appeared in the blood coincident with recovery, and has persisted, in gradually decreasing amounts, for four and one-half months.

Patient St. (See Chart 2). History Number 48,419. White, female, age 22 years. Admitted: March 26, 1933. Disease: Erysipelas of the face.

Résumé of clinical course. The acute condition which brought this patient to the hospital was obscure. She had been exposed to mumps and on entry there was a slight degree of tender swelling at the angle of each jaw. There was some soreness of the throat. Whether the swellings were parotid glands or lymph 
glands was not clear. After two days the temperature became normal, the condition subsided and patient seemed well. Six days later she had a shaking chill and her temperature suddenly rose to $104^{\circ} \mathrm{F}$. She became acutely intoxicated, developed hyperesthesia of forehead and scalp, and the tender swelling at the angle of the right jaw returned. Twenty-four hours later it was evident that she had erysipelas, and the lesion spread rapidly over the forehead, nose, eye-

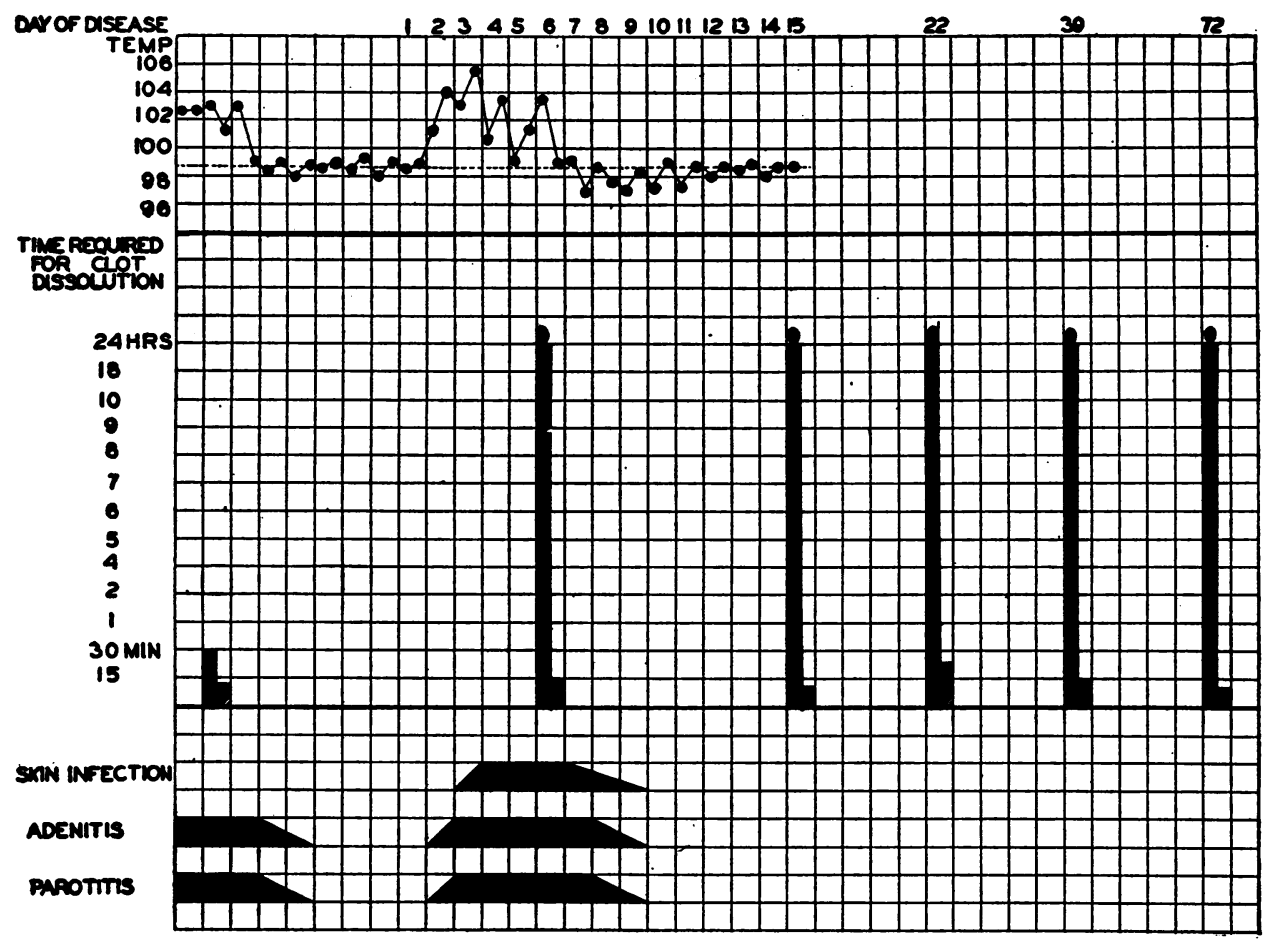

Chart 2. Patient St., Female, Age 22 Years. Disease, Erysipelas

lids and face down to the root of the ears. Recovery abruptly occurred five days after sudden onset.

Laboratory findings. White blood cells, 20,000 per c.mm. on admission. 18,000 per c.mm. at time of $2 \mathrm{~d}$ febrile reaction. Blood cultures : Sterile. Throat culture: Few colonies of hemolytic streptococci present on admission and during erysipelas.

Fibrinolytic test. One specimen of blood was obtained from this patient at the time of the first undiagnosed febrile episode. The rate of fibrin liquefaction was only slightly longer than that of the normal control; 25 minutes as compared to 14 minutes. A second specimen of plasma procured on the day before the temperature suddenly became normal and recovery set in, was resistant to the maximum degree. The blood of this patient has retained maximum resistance at each test, the last of which was performed three months after recovery. 
Comment. In this case of erysipelas, maximum anti-fibrinolysis appeared in the blood coincident with recovery from an acute infection of 5 days duration. Insusceptibility to streptococcal fibrinolysis has persisted for three months.

Patient La. (See Chart 3A). History Number 48,508. Male, colored, age 32 years. Admitted: March 30, 1933. Disease: Erysipelas of the face.

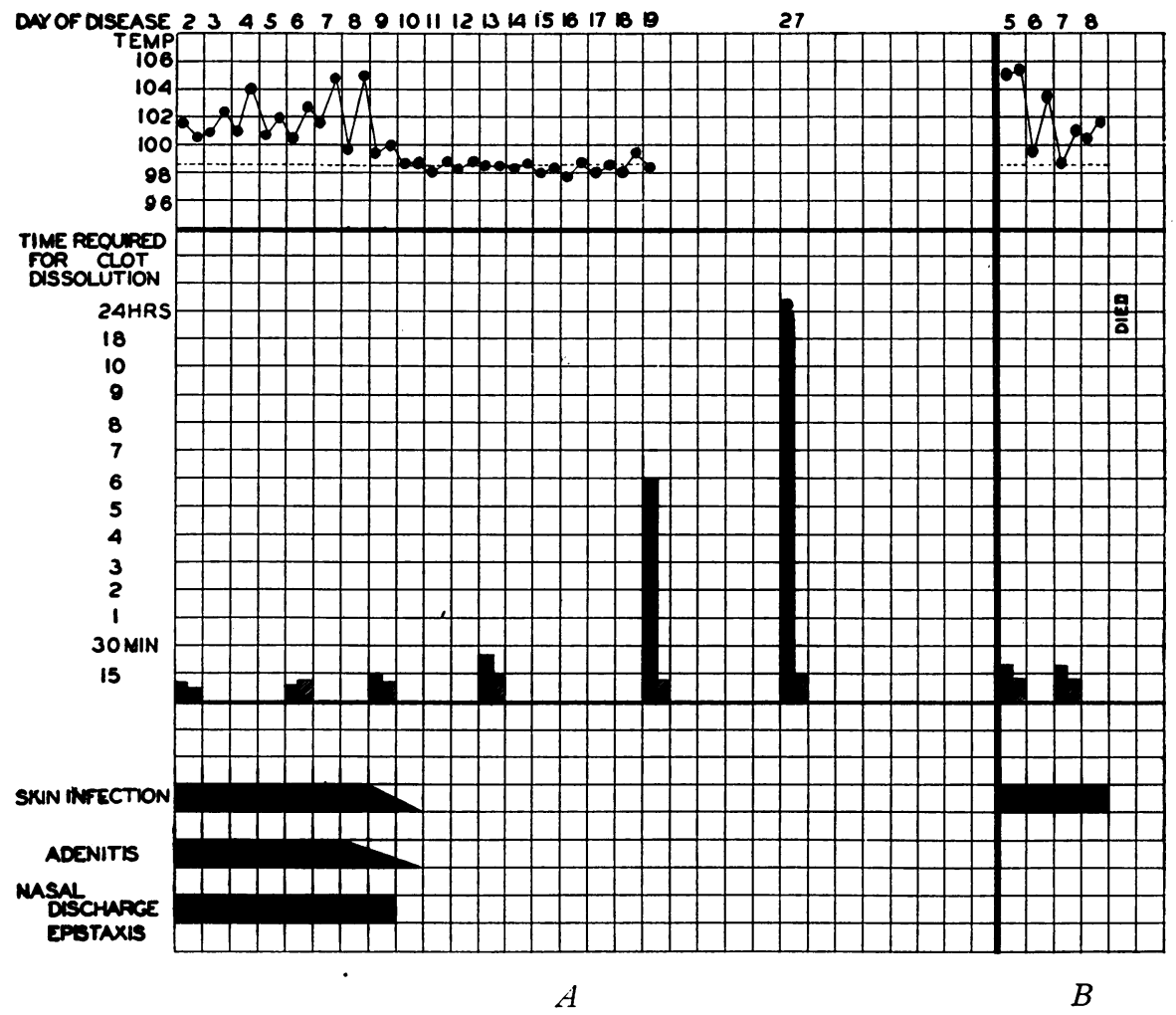

Chart $3 A$. Patient La., Male, Age 32 Years. Disease, Erysipelas 3B. Patient Hu., Male, Age 72 Years, Disease, Erysipelas

Résumé of clinical course. The patient was admitted on the second day of disease. Onset was sudden on the day before admission with the development of sore throat, weakness, chills and fever, and headache. The next day his face became swollen. purulent nasal discharge appeared and cervical glands were enlarged and tender. He came to the hospital later on this same day. On admission he was moderately ill, and had the typical lesion of facial erysipelas involving the skin over the bridge of his nose and malar eminences. There was nasal obstruction from the discharge, and tonsils were greatly swollen but not acutely inflamed. Cervical glands were large and tender. 
Laboratory findings. White blood cells, 11,720 per c.mm. Blood culture: Sterile. Nasal culture: Many colonies of hemolytic streptococci. Throat culture: Few colonies of hemolytic streptococci.

The erysipelas lesion spread in the usual manner over the entire face, involved ears and mastoid regions, and terminated with a drop in temperature on 9 th day after onset. Nose bleeds were frequent during the acute illness. Convalescence was uneventful.

Fibrinolytic tests. In tests made with the first four samples of plasma from this patient, no evidence of resistance to fibrinolysis was demonstrable. The results are of interest since the third specimen was obtained on the day of recovery, and the fourth specimen was procured on the fourth day after recovery. Anti-fibrinolysis first appeared ten days after recovery, and, even then, the clot liquefied in six hours. A sample of blood taken on the 19th day of convalescence remained solid for 24 hours. It is not possible to state when maximum resistance was acquired, since no blood was taken between the 10th and 19th day of convalescence.

Comment. Resistance became demonstrable in this case of erysipelas late in the disease. In the other two patients with erysipelas maximum resistance was present on the day of recovery.

Patient Hu. (See Chart 3B). History Number 48,391. White, male. age 72 years. Admitted: March 23, 1933. Disease: Erysipelas of the face.

Résumé of clinical course. The patient was admitted on the sixth day of disease, which followed a severe blow on the head. He was knocked unconscious and his face was lacerated. The infection began at the wound, and spread over his face and scalp.

On admission he was critically ill and very weak. His face, eyes, and scalp were greatly swollen. He was dyspneic and there was marked arteriosclerosis.

Laboratory findings. White blood cells, 8,500 per c.mm. Blood culture: Sterile. Wound culture: Hemolytic streptococci.

The patient died suddenly three days after admission.

Fibrinolytic tests. Two specimens of blood were obtained from this patient during his acute illness. In both instances the plasma-clot was rapidly liquefied.

Comment. The case is an example of fatal outcome of erysipelas in an individual, age 72 years, whose blood did not contain demonstrable amounts of anti-fibrinolytic substances.

Patient Qu. (See Chart 4). History Number 47,965. White, female, age 20 years. Admitted : February 22, 1933. Disease : Scarlet fever.

Résumé of clinical course. Patient was admitted on second day of disease. Onset was sudden on the day before admission with the development of malaise, sore throat, chill and fever. Rash appeared during the night.

On admission patient was only mildly intoxicated although the throat was fiery red, and typical scarlatinal rash was present over entire body. 
Laboratory findings. White blood cells, 16,600 per c.mm. Blood culture: Sterile. Throat culture: Many colonies of hemolytic streptococci.

The patient's symptoms were never severe, her rash faded on the third day, and her temperature, which was never high, became normal on the fifth day. She had vague arthralgia and myalgia. Desquamation began on the fourth day.

Convalescence was normal until the thirteenth day of recovery when she developed tachycardia. The next day her temperature rose to $101.6^{\circ} \mathrm{F}$. Cervical glands became moderately enlarged and tender. Four days later the fever disappeared, pulse rate slowed, and the glands subsided. Convalescence was then uneventful.

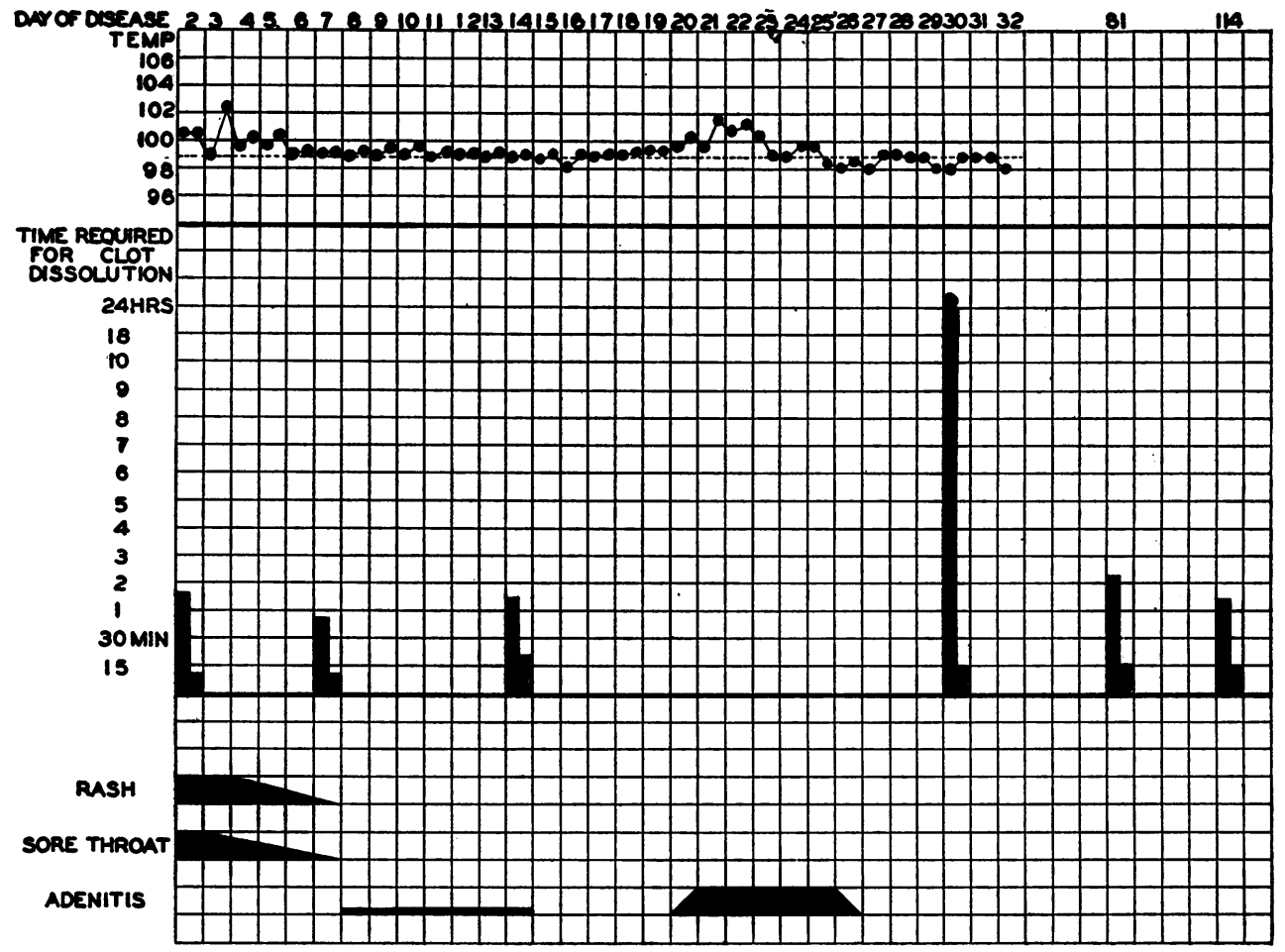

Chart 4. Patient Qu., Female, Age 20 Years. Disease, Scarlet Fever

Fibrinolytic tests. The fibrin clot from the first sample of blood, taken on the day of admission, required one hour and thirty minutes to dissolve although the normal control liquefied in 12 minutes. With blood procured on the second day of normal temperature, there was only slight difference between the behavior of the patient's plasma and normal plasma. No change in dissolution time was noted in blood taken seven days later. However, maximum resistance was present in the fibrin-clot of blood taken on the 25 th day after recovery from scarlatina ( 5 days after the late febrile episode). With bleedings about 2 and 3 months later, the degree 
of resistance had greatly diminished, although the rate of dissolution was longer than the normal control.

Comment. In this case of scarlet fever, maximum resistance was not demonstrable on the second or seventh days of convalescence; but appeared in the sample of blood taken 25 days after recovery, and diminished definitely during the next 3 months.

Patient Os. (See Chart 5). History Number 48,327. White, female, age 20 years. Admitted: March 19, 1933. Disease: Scarlet fever. Treated with scarlatinal antitoxin (2 doses of 6,000 units each, one intramuscularly, and one intravenously).

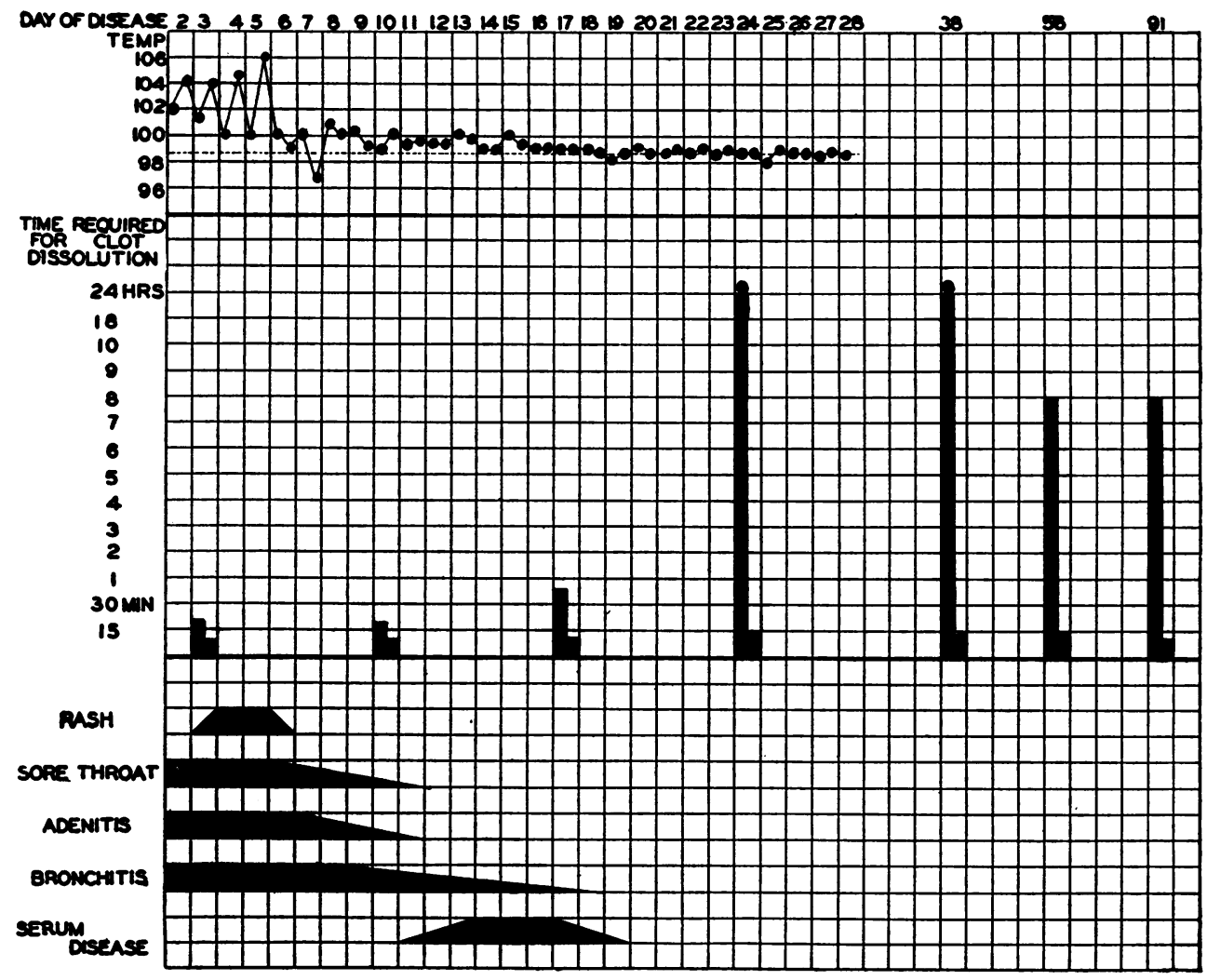

Chart 5. Patient Os., Female, Age 20 Years. Disease, Scarlet Fever (Treated with Scarlatinal Antitoxin)

Résumé of clinical course. Patient was admitted on second day of disease. Onset was accompanied by sore throat, malaise, and fever. On admission she appeared acutely intoxicated and her throat, which was acutely inflamed, was very painful. The cervical glands were enlarged and tender. There was a faint erythematous blush in both axillae. 
Laboratory findings. White blood cells, 9,800 per c.mm. Blood culture: Sterile. Throat culture: Many colonies of hemolytic streptococci.

Nausea and vomiting developed during the first day, and on the next day a typical scarlatinal rash covered most of the body. She received 6,000 units of antitoxin intramuscularly. On the following day, since no improvement was noticeable, a second injection of 6,000 units of antitoxin was given intravenously. During the next 24 hours, after a chill, the temperature reached a level only slightly above normal, and the rash very quickly faded. Her symptoms were greatly improved.

Eight days later serum sickness developed which lasted about six days. The attack of scarlet fever was not followed by any complications.

Fibrinolytic tests. With the first 3 tests on the blood from this patient, anti-fibrinolytic properties were not demonstrable. The initial bleeding was obtained during acute illness; the other two were taken on the fourth and eleventh days after recovery. Maximum resistance appeared on the 18th day of convalescence, and was maintained to the $32 \mathrm{nd}$ day. Two subsequent tests on the 56th and 89th days, respectively, still showed marked resistance, although less than maximum.

Comment. In this case of scarlet fever, as in the preceding one, insusceptibility to fibrinolysis did not become demonstrable until late in convalescence.

Patient Ow. (See Chart 6). History Number 48,425. White, female, age 21 years. Admitted: March 26, 1933. Disease: Scarlet fever. Acute sinusitis. Treated with scarlatinal antitoxin (1 dose intramuscularly of 6,000 units).

Résumé of clinical course. Admitted on first day of disease, moderately ill with fever, sore throat and beginning rash.

Laboratory findings. White blood cells, 11,400 per c.mm. Blood culture: Sterile. Throat culture: Many colonies of hemolytic streptococcus.

During the next 24 hours the rash intensified, and intoxication became marked. Six thousand units of antitoxin were given intramuscularly. The rash faded slowly in the next few days, but the acutely inflamed throat continued to be very painful. On the sixth day she developed signs of acute right maxillary sinusitis. Three days later irrigation of the sinus yielded a moderate amount of thick pus. An abundant growth of Streptococcus viridans in pure culture was obtained from the pus. The sinus infection required repeated irrigations and persisted during her stay in the hospital. Other paranasal sinuses were less severely infected. No cultures were obtained from them. Enlarged and tender cervical glands persisted during her illness.

On the 9th day she developed mild serum sickness which disappeared after 5 days.

She was discharged from the hospital with sinus infection still active. Under continual treatment, the infection of sinus has gradually improved. A culture of the discharge from the sinus taken after the patient left the hospital, yielded many colonies of hemolytic streptococci.

Fibrinolytic tests. A sample of this patient's blood taken on the first day of illness, supplied a fibrin-clot which liquefied rapidly. However, the 


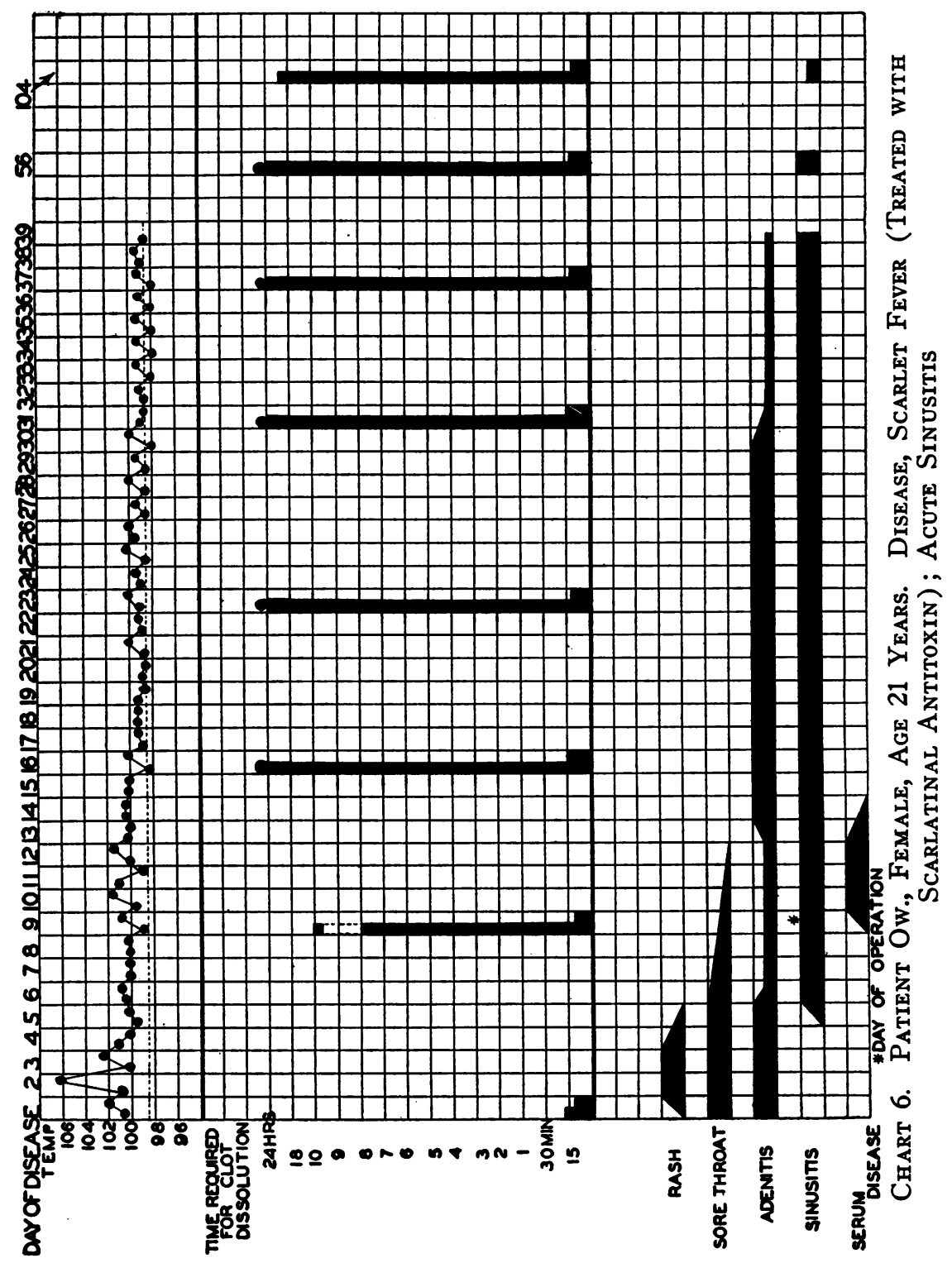


fibrin-clot of a bleeding taken 8 days later-3 days after the first evidence of acute sinusitis, and on the day when thick pus was drained from the sinus-required 8 to 18 hours for liquefaction. A third specimen procured a week later remained solid for 24 hours. This maximum resistance has been maintained up to the time of the last test when liquefaction was complete in 20 hours.

Comment. The development of resistance in this case came earlier than in the other two uncomplicated cases of scarlet fever, and has been maintained to a greater degree for a longer period of time. In this connection it is interesting to note that the sinus infection has required continual treatment and remains as a low grade chronic infection. The appearance of resistance in association with the development of purulent complication is of interest. That the organism in the purulent discharge from one sinus was Streptococcus viridans is also of interest, since throat cultures yielded hemolytic streptococci. Cultures from other less severely infected paranasal sinuses were not obtained. A subsequent sinus culture was positive for hemolytic streptococci.

Patient Ba. (Chart 7). History Number 48,074. White, female, 23 years. Admitted: March 1, 1933. Disease: Acute tonsillitis (Hemolytic streptococcus).

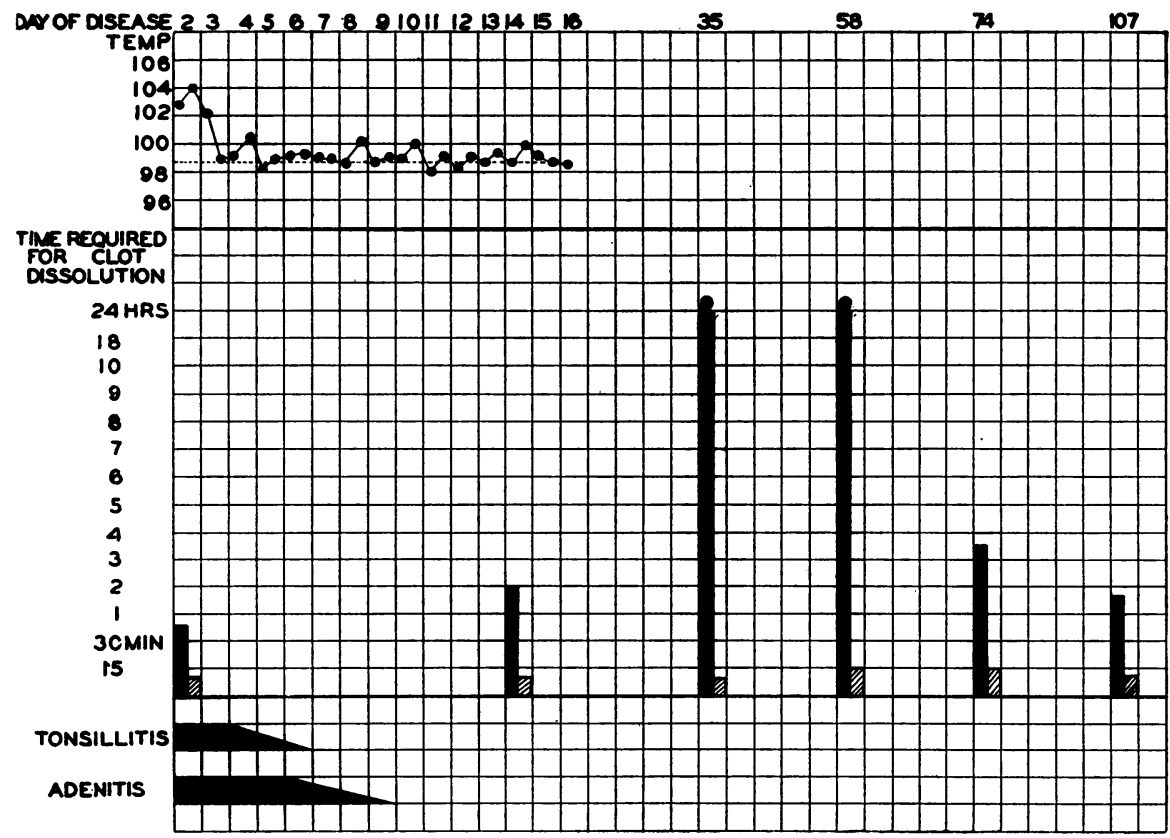

Chart 7. Patient Ba., Female, Age 23 Years. Disease, Acute TONSILLITIS 
Résumé of clinical course. Patient was admitted to the hospital on the second day after the sudden development of acute sore throat.

Laboratory findings. White blood cells, 16,800 per c.mm. Throat culture: Many colonies of hemolytic streptococci.

The throat was very acutely inflamed, and the tonsils were spotted with yellowish-white exudate. Swallowing was painful. General intoxication was marked. Cervical glands were large and tender.

Temperature became normal on the third day, and symptoms subsided rapidly. There were no complications.

Fibrinolytic tests. Repeated tests with the plasma-clot from this case of uncomplicated acute hemolytic streptococcus tonsillitis show that, although slight delay in fibrinolysis was evident in a sample of blood taken 8 days in convalescence, maximum resistance was first demonstrable on the 35 th day after recovery. Insusceptibility to dissolution endured for 3 weeks, but subsequent tests showed a rapid diminution of resistance, which 4 months after the acute illness was only slightly manifest.

Comment. In this case of acute tonsillitis, as in the two patients with uncomplicated scarlet fever, resistance was demonstrable late in convalescence. Since no observations were made between the 13th and 39th day, the exact time of development of resistant properties is undetermined. The duration of acute illness was only four days.

Patient Hi. (Chart 8). History Number 43,979. White, female, age 26 years. Admitted: March 12, 1933. Disease: Acute tonsillitis (Hemolytic streptococcus).

Résumé of clinical course. The patient, admitted on the second day after abrupt onset, had a mild attack of acute tonsillitis. Tonsils were inflamed and edematous. Cervical glands palpable and slightly tender.

Laboratory findings. White blood cells, 8,750 per c.mm. Throat culture: Many colonies of hemolytic streptococci.

After two days in bed, recovery was complete. There were no complications.

Fibrinolytic tests. In this patient, a test with the plasma-clot procured on the 7 th day of convalescence was not significantly different from the first test. With blood taken on the 22 nd day of convalescence fibrinolysis required 8 hours. Subsequent observations, on the 43rd, 59th and 109th days, showed a persistence of resistance at a lower level.

Comment. This patient had a mild attack of uncomplicated acute tonsillitis of short duration. Resistance appeared late in convalescence and was never maximum.

Patient Fu. (Chart 9). History Number 48,423. White, female, age 21 years. Admitted: March 25, 1933. Disease: Acute pharyngitis (Hemolytic streptococcus). Acute maxillary sinusitis.

Résumé of clinical course. Patient was admitted to the hospital on the second day of disease, complaining of sort throat and headache. She was 


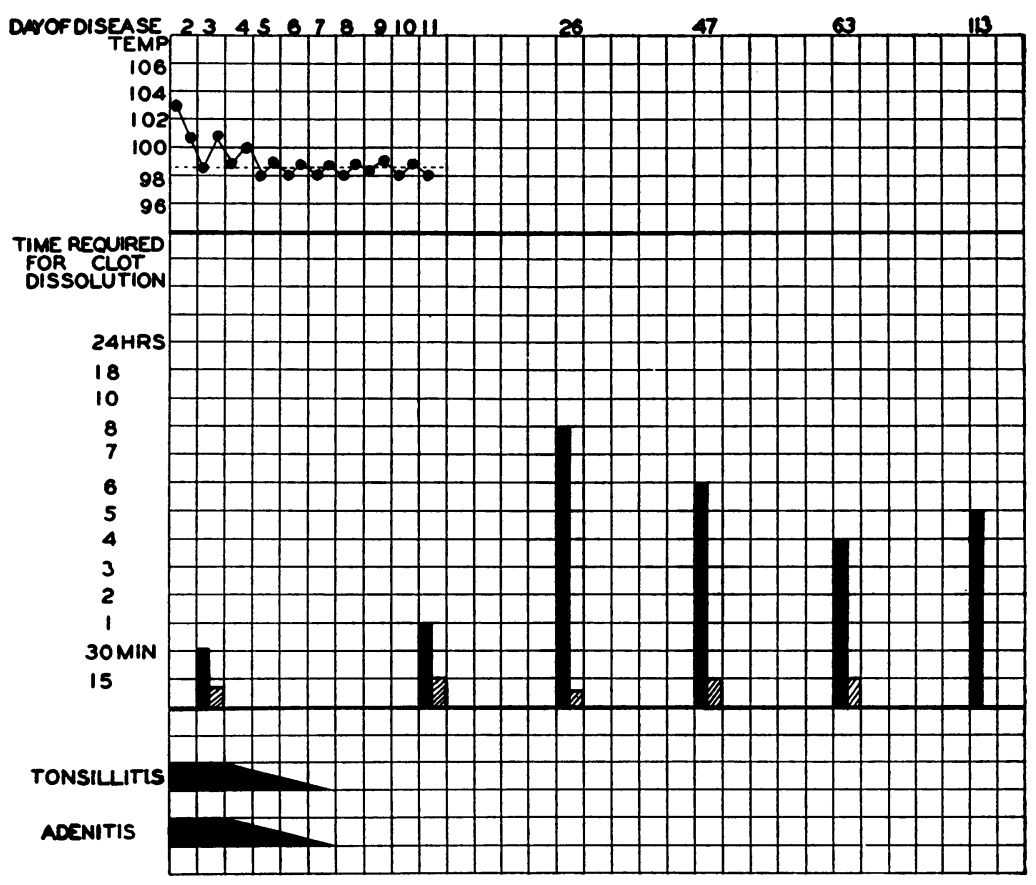

Chart 8. Patient Hi., Female, Age 26 Years. Disease, Acute TONSILLITIS

moderately ill; her throat was acutely inflamed, and there was tenderness over the left antrum.

Laboratory findings. White blood cells, 14,500 per c.mm. Throat culture: Few colonies of hemolytic streptococci.

Two days after admission the pain in the left antrum became very severe, and after two days of ineffectual local treatment, the sinus was irrigated. Bloody mucoid exudate was discharged. Culture of pus yielded only pneumococcus (Group IV).

Convalescence was rapid and uneventful. The sinus infection subsided after drainage and did not return.

Fibrinolytic tests. The first sample of plasma from this patient was definitely resistant. It was obtained on the 3rd day of acute pharyngitis which was complicated by acute sinusitis. Nine days later a fibrinolytic test showed an additional increase in resistance. Three weeks later resistance had decreased but was still marked. One and one-half months and three months after the acute illness, fibrin-clot from the patient's plasma was completely susceptible.

Comment. Resistance was manifest early in this patient. Symptoms of sinusitis appeared very quickly after the beginning of the throat infection. The sinusitis healed readily, and resistance disappeared more quickly than in the other patients. The isolation of pneumococcus from the in- 


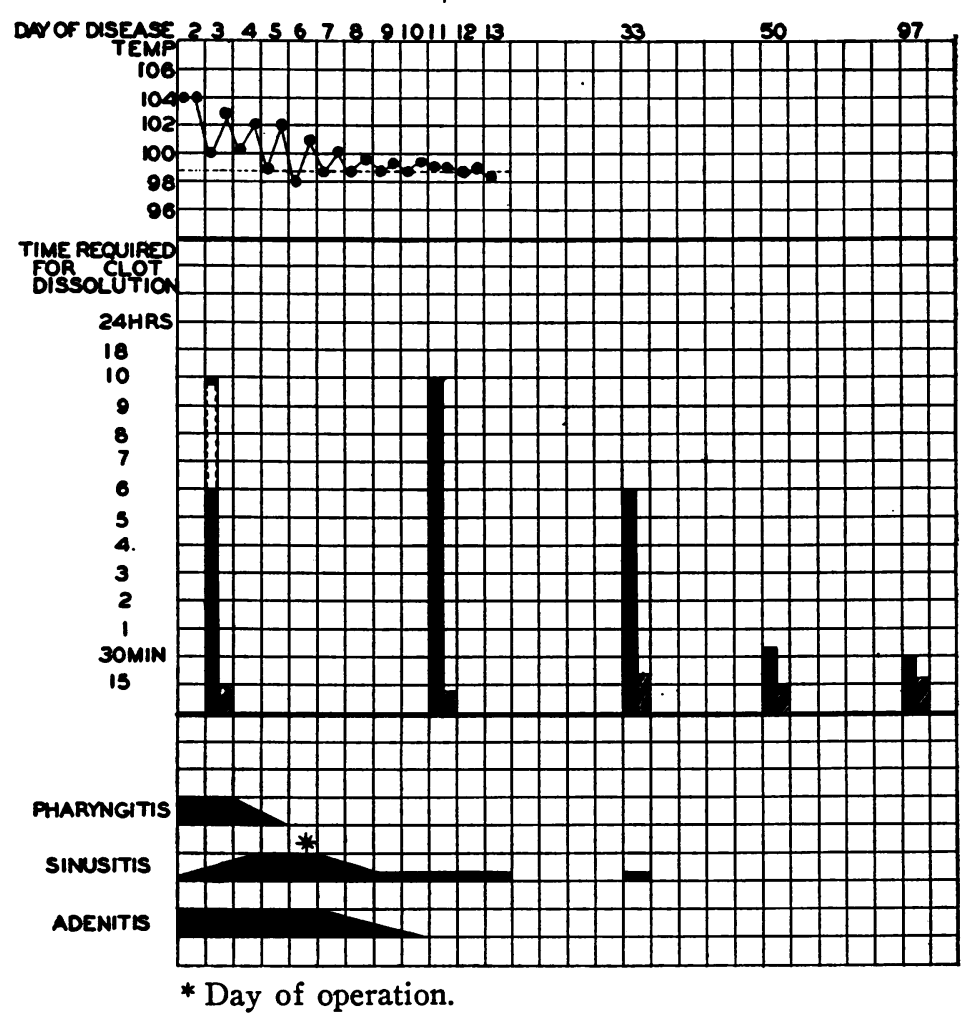

Chart 9. Patient Fu., Female, Age 21 Years. Disease, Acute Pharyngitis; Acute Sinusitis

fected antrum is of interest, in view of the fact that hemolytic streptococci were present in the throat.

Patient Ch. (Chart 10). History Number 48,745. White, female, age 21 years. Admitted: April 13, 1933. Disease: Acute maxillary sinusitis, bilateral (Hemolytic streptococcus). Acute otitis media, bilateral (Hemolytic streptococcus). Acute pharyngitis (mild) (Hemolytic streptococcus).

Résumé of clinical course. The patient was admitted to the hospital on the 5th day of an acute febrile upper respiratory tract infection with involvement of both maxillary sinuses on the third day of disease, and acute right otitis media on the fourth day of disease. The illness began with pharyngitis which was never severe, and was not prominent on admission. Exquisite tenderness and pain over the sinuses and in the ears constituted the chief complaint. Right myringotomy was performed on the first day in the hospital with the evacuation of thin serosanguineous exudate. Later in the same day, left ear became painful, and the ear drum was incised; thin pus was obtained. Both antra, which were acutely infected, were also irrigated. 
Cultures of pus from both ears and both antra yielded abundant bacterial growth, which was predominently hemolytic streptococcus.

Laboratory findings. White blood cells, 17,200 per c.mm.

Although adequate discharge of pus relieved the severity of symptoms, fever persisted for 12 days. When the patient left the hospital, the ears and sinuses had apparently completely healed.

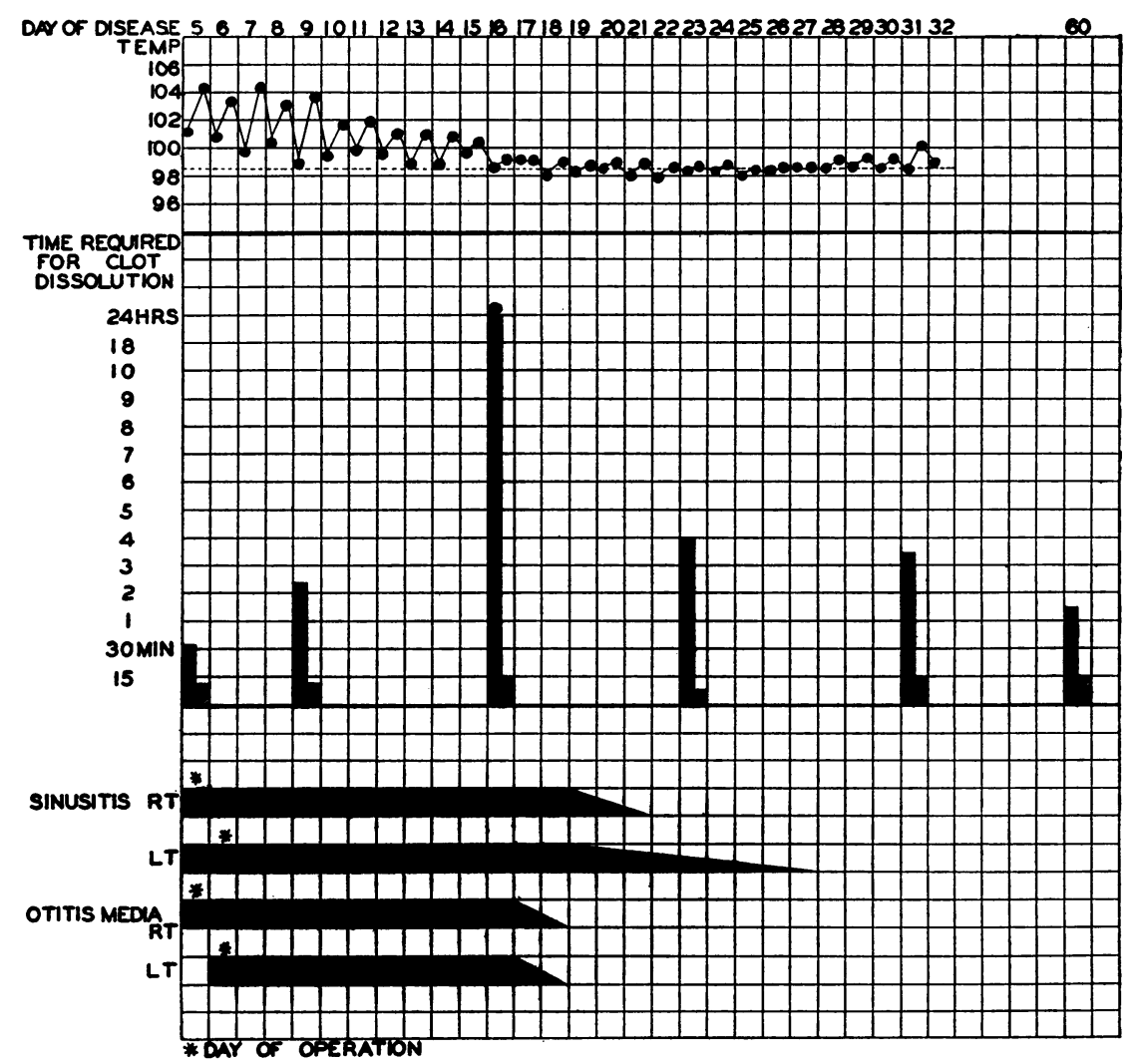

Chart 10. Patient Ch., Female, Age 21 Years. Disease, Acute Sinusitis; Acute Otitis Media

Fibrinolytic tests. Tests with the blood of this patient, who had multiple purulent inflammations, reveal that, early in the active disease, fibrinolysis proceeded rapidly. Four days later, delay in liquefaction was definite but not marked. No additional tests were taken until the 16th day, at which time anti-fibrinolysis was maximum. From this point on, subsequent tests showed progressive diminution in delayed liquefaction.

Comment. The appearance of maximum resistance to fibrinolysis occurred in the blood of this patient with purulent complications of upper respiratory infection at a time when the condition was subsiding. Complete insusceptibility did not seem to appear quite as abruptly as in the other 
cases with suppuration; but it was demonstrable earlier than in the blood of patients without complications. Recovery from infection was complete in about 3 weeks and marked resistance was not maintained for a long time.

Patient V.H. (Chart 11). History Number 40,215. White, female, age 24 years. Admitted : February 27, 1933. Disease : Acute otitis media (Hemolytic streptococcus).

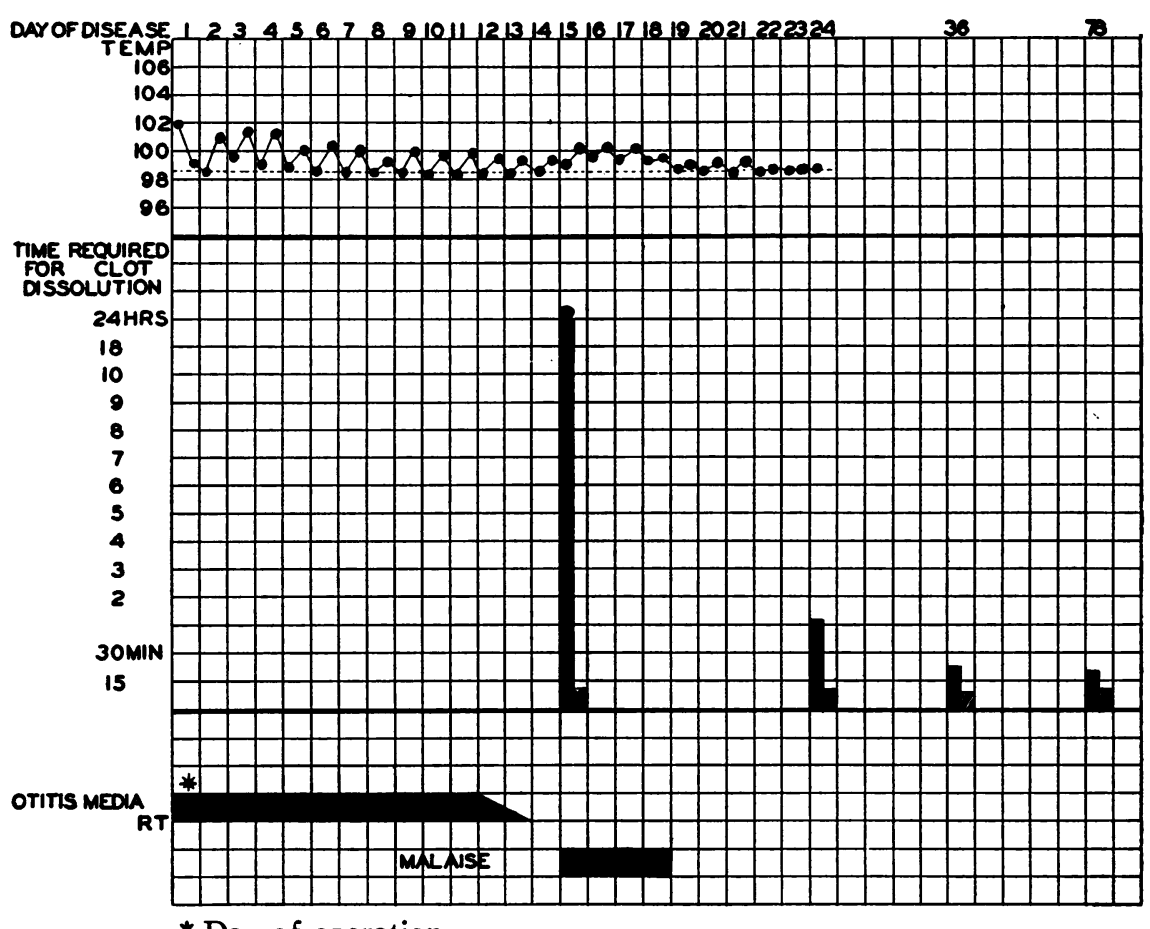

Chart 11. Patient Vh., Female, Age 24 Years. Disease, Acute Otitis Media

Résumé of clinical course. After two days of a mild nasopharyngitis, patient developed suddenly acute severe pain in right ear. She was admitted to the hospital this same day and myringotomy was performed at once. Thin serosanguineous fluid was evacuated.

Laboratory findings. White blood cells, 5,000 per c.mm. Later 13,500 per c.mm. Ear culture: Heavy growth of pure culture of hemolytic streptococci. Throat culture: Heavy growth of pure culture of hemolytic streptococci.

Adequate drainage was difficult to maintain, and three subsequent myringotomis were necessary. Pain in the ear and temperature gradually subsided in about a week. On the 13 th day temperature became slightly elevated again, and she had general malaise and aching. After 3 or 4 days, the symptoms subsided, and recovery was uneventful. At the time of discharge from the hospital the ear had stopped draining. There was slight impairment to hearing. 
Fibrinolytic tests. Unfortunately, tests were not done early in this patient's illness. It is, therefore, impossible to state whether the patient's plasma-clot was susceptible to dissolution at the beginning. The first test, which was made with a sample of blood taken on the 15th day showed maximum resistance. Three subsequent tests during the 2 months after discharge from the hospital showed that resistance had disappeared completely.

Comment. The results of fibrinolytic tests with the blood of this patient exemplifies the presence of maximal resistance in a person with suppurative hemolytic streptococcus infection, and the subsequent rapid disappearance of anti-fibrinolytic properties from the blood following complete recovery.

In all the cases of acute streptococcus disease, with which Charts 1 to 11 are concerned, it may be seen that convalescence is, in every instance, attended by the presence in the patients' plasma-clot of maximum or very marked resistance to the fibrinolytic property of hemolytic streptococci. That fibrin from the same individuals, obtained before and after recovery, changed from susceptible to maximally resistant in a short period of time evidences the fact that infection with hemolytic streptococci stimulates the production of anti-fibrinolytic substances. Resistance was equally marked in the blood of patients convalescent from erysipelas, scarlet fever, and acute tonsillitis, with or without complications.

The duration of anti-fibrinolytic resistance has varied but seems to be influenced by the nature and length of time that active infection persists. Individual reactivity, also, undoubtedly participates in the degree and extent of this mechanism as in other immunological responses.

The number of cases of each type of infection is too small to attach undue significance to the time in the course of the disease at which resistance became marked. The following résumé serves, however, as a summary.

Two cases recovered from erysipelas developed maximal resistance on the day of recovery. (Charts 1 and 2.)

One case recovered from erysipelas developed marked resistance 7 days after recovery, which was found to be maximum 8 days later. (Chart 3.)

One fatal case of erysipelas failed to develop resistance. (Chart 3.)

Two cases recovered from uncomplicated scarlet fever developed maximal resistance on the 17th and 24th days respectively. (Charts 4 and 5.)

One case of scarlet fever, complicated by purulent sinusitis on the 4th day, developed resistance on the 6th day. (Chart 6.)

Two cases recovered from acute tonsillitis without complications were maximally resistant in tests done on the 26th and 35th days of disease. (Charts 7 and 8.)

Three cases recovered from acute tonsillitis with suppurative complications exhibited marked or maximum resistance in tests done on the $3 \mathrm{~d}$, 
15th, and 16th days respectively after the appearance of purulent infections. (Charts 9,10 and 11.)

In the 8 cases of upper respiratory streptococcal infection, the development of resistance appeared later in the 4 uncomplicated cases than it did in the 4 patients with purulent complications. Whether or not a purulent inflammatory process hastens this immune response cannot yet be finally stated. However, the delayed anti-fibrinolytic response in some instances, and the immediate response in others, forms a striking part of the results of this investigation.

Repeated fibrinolytic tests with plasma-clot of blood from patients, before and after recovery from other infections

In order to determine whether or not resistance to the fibrinolytic activity of hemolytic streptococci is specifically induced in streptococcus infections, a series of repeated control tests have been performed with plasma from cases of other acute diseases.

The results with eleven of these patients is contained in Charts 12 to 15 inclusive. The infections from which the patients suffered are as follows:

Five cases of pneumococcus pneumonia; 1 case of typhoid fever; 1 case of gonococcal arthritis; I case of active pulmonary tuberculosis ; I case of malaria (Therapy for general paresis); 1 case of acute gangrenous appendicitis (Colon bacillus) ; 1 case of abscess of muscle (gram positive, anaerobic bacillus).

In each of these patients the clinical and bacteriological diagnosis was definite; hemolytic streptococcus infection was ruled out. Except for one case, a detailed account of the clinical course is unnecessary.

In Chart 12, the results of repeated fibrinolytic tests with the blood of four cases of pneumococcus pneumonia are given.

Case Th. (History Number 49,201. Admitted: May 11, 1933) had lobar pneumonia due to Type VII pneumococcus. He recovered uneventfully.

Case Ja. (History Number 49,035. Admitted: April 30, 1933) had lobar pneumonia due to Type IV pneumococcus. He recovered without complications.

Case Na. (History Number 46,954. Admitted: December 18, 1932) had Type III pneumococcus pneumonia, with uncomplicated recovery.

Case Ha. (History Number 49,572. Admitted: May 31, 1933) had an overwhelming Type I pneumococcus septicemia at the time of full term pregnancy, and died on the 6th day of disease in spite of anti-pneumococcus serum therapy.

From Chart 12 it may be seen that clot liquefaction occurred rapidly with each sample of blood taken either before or after recovery. In Case Th., the last test was made with plasma-clot obtained on the 40th day after recovery. Case Na. was repeatedly tested at intervals for 33 days. Case Ja. was not available after the 19th day; only one specimen of blood was obtained from the fatal case, Case Ha. 
PATIENT: TH.T DISEASE: LOEAF RNEUMONIA

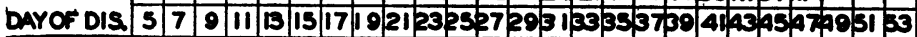

10

102.

100

98

\section{FIBRINOLVSIS}

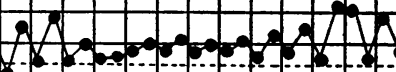
1

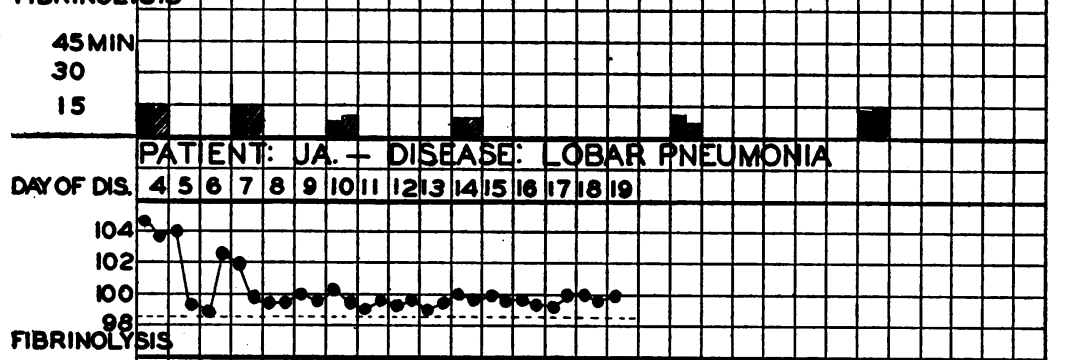

FIBRINOLYSIS

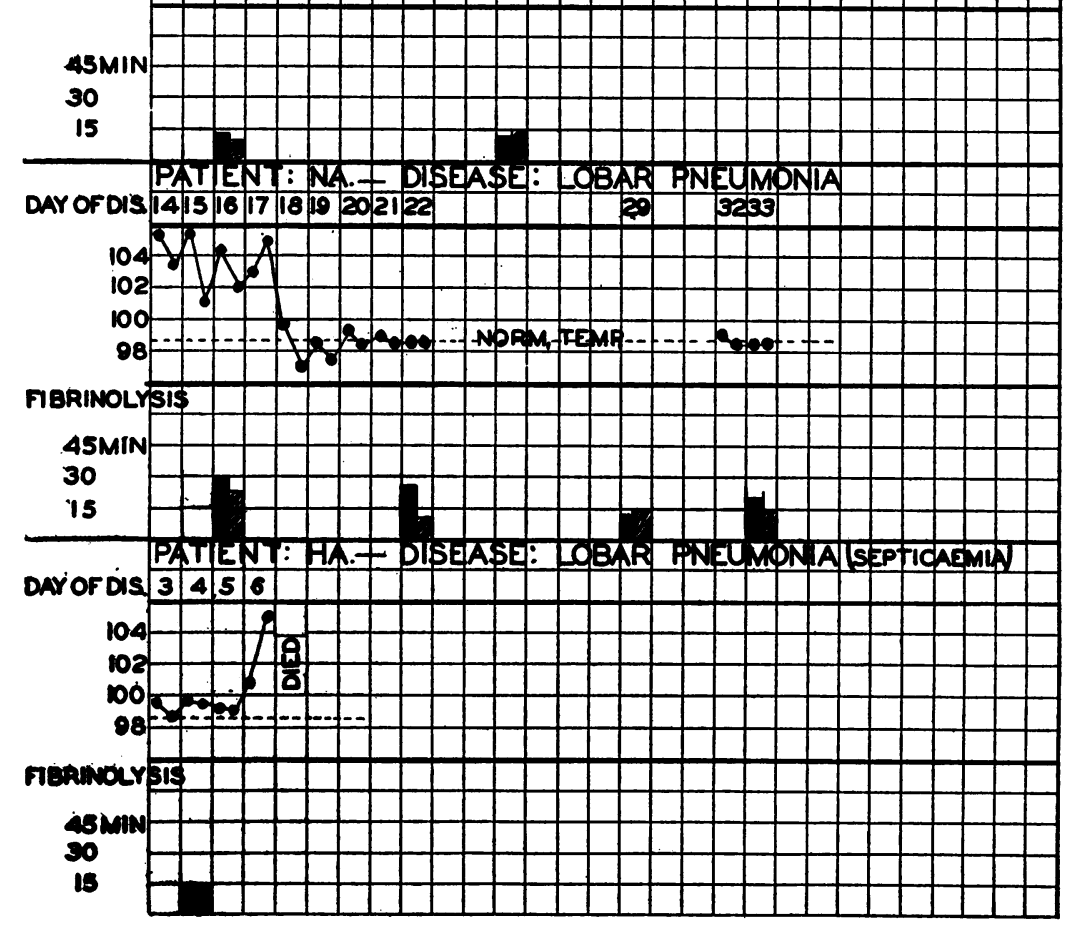

Chart 12. Patients Th., Ja., Na., and Ha.

The fifth case of pneumonia, Case $\mathrm{Pa}$., presented results which are unique and have not been noted in any other individuals. Although no explanation can be offered at the present time, the change in reactivity of the patient's blood was sufficiently striking to justify presentation. 
Case Pa. (See Chart 13). (History Number 49,363. Admitted: May 25, 1933.) The patient was admitted to the hospital seriously ill with Type I pneumococcus pneumonia and septicemia. He was treated vigorously with concentrated anti-pneumococcus serum and recovered without complications. It is interesting to note that 2 months before admission he had trouble with his right ear which was followed by paralysis of some of the right cranial nerves. Because of the fact that he was known to have syphilis, the neurological condition was thought to be recurrent neural syphilis in an individual who had received inadequate treatment. That his spinal fluid was normal, however, threw doubt on the correctness of the suggested diagnosis.

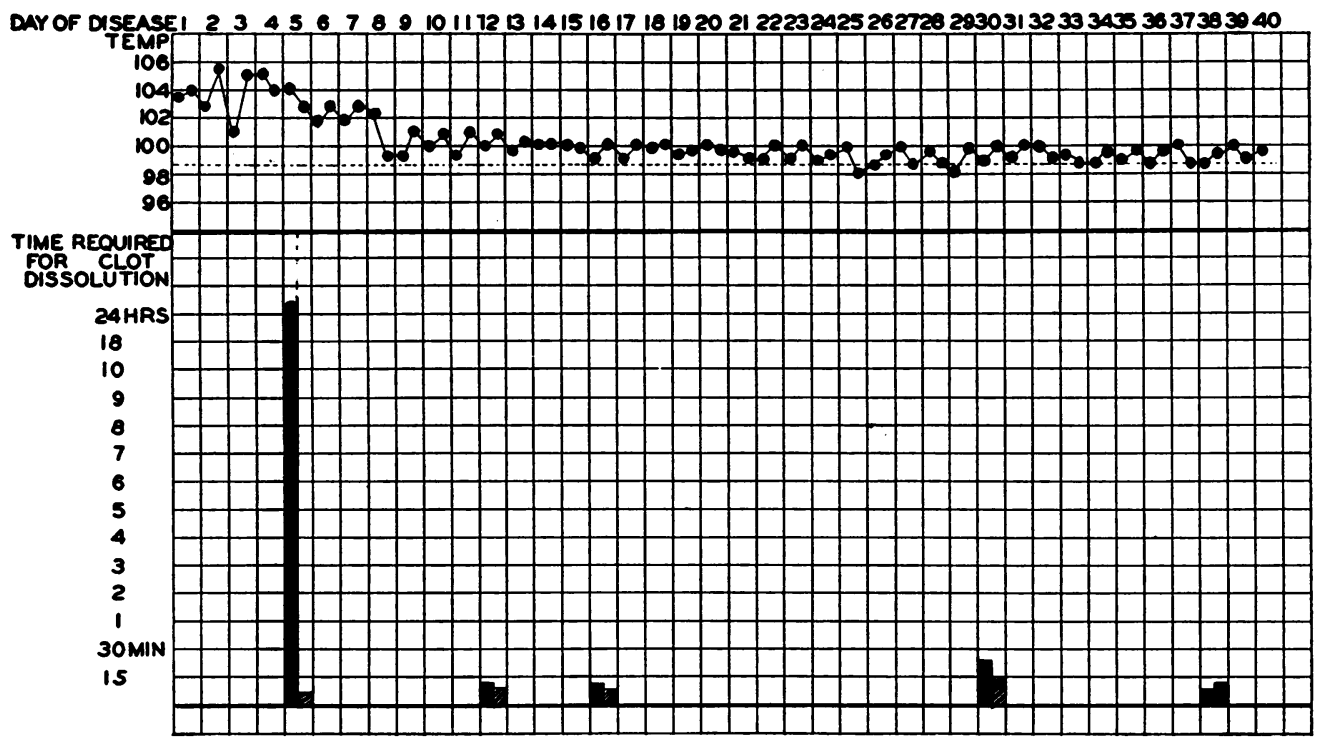

Chart 13. Patient Pa. Disease, lobar Pneumonia (Type I Septicemia) (Serum Therapy)

From the patient's chart it may be seen that the fibrin clot from the sample of blood taken on admission was totally resistant to fibrinolysis (Chart 13). Seven days later, the anti-fibrinolytic properties had entirely disappeared. Furthermore, in all bleedings for the next 4 weeks, no return of resistance was demonstrable.

The presence of maximum resistance in this patient's blood at the time of early severe illness has not been encountered in any of 8 other cases of pneumonia, whose blood was tested during the acute disease. That the initial resistance might have resulted from the infection of the ear two months previously is a possibility. The abrupt disappearance of resistance is inexplicable at the present time, and one can only speculate as to the effect which large amounts of anti-pneumococcus serum might have on the inhibiting properties of the blood. The last bleeding in this patient was taken 31 days after recovery. 
In Chart 14 the results with three other cases of different types of infection are presented.

Case Sw. (History Number 48,821. Admitted: April 18, 1933) had acute gonococcal arthritis with multiple joint involvement. Gonococci were present in purulent exudate obtained from the joints.

Case Ca. (History Number 48,929. Admitted: April 24, 1933) had active pulmonary tuberculosis. The sputum contained many tubercle bacilli.

Case An. (History Number 36,328. Admitted: May 14, 1933) had typhoid fever. Typhoid bacilli were present in the blood stream and also in the stools.

The plasma-clot from the blood of each of these three patients was liquefied in every test by active cultures in less than one hour. By following the cases with repeated bleedings for at least 30 days, no change in dissolution time was noted. That the plasma-clot from patients acutely ill liquefies at a slightly slower rate than that of the susceptible normal control, has been repeatedly observed.' However, the rate of dissolution occurring with the blood of the three cases presented above belongs to the group designated susceptible.

Three additional cases of infection, with whose blood repeated fibrinolytic tests have been performed, are recorded in Chart 15.

Case Sc. (History Number 49,333. Admitted: May 19, 1933) had general paresis and was admitted to the hospital for therapeutic malarial infection. The intermittent fever indicates the time of active malaria, which was terminated by quinine therapy. Blood was obtained before, during, and after malarial inoculation.

Case Bo. (History Number 49,751. Admitted: June 13, 1933) had an acute cellulitis of the leg, which, after several days of palliative treatment, was incised. A gram positive anaerobic bacillus was isolated from the pus. No hemolytic streptococci were present. Blood was taken before and after operation.

Case $\mathrm{Cr}$. (See Chart.) (History Number 49,284. Admitted: May 13, 1933) had acute appendicitis. At operation the appendix was found to be gangrenous, and there was some localized peritonitis. Drainage was carried out for several days and recovery was uneventful. Colon bacilli were obtained from the pus. The first sample of blood was obtained after operation.

From Chart 15 it may be seen that resistance is absent from the blood of these three patients and that repeated tests did not reveal any change in the rate of dissolution during the period of observation.

The results of fibrinolytic tests with the plasma-clot of blood from the cases of acute illness just described indicate that resistance to streptococcal fibrinolysis is not a general reaction to infection. In ten of the selected cases with whose blood repeated tests were made, the rapid rate of fibrinolysis did not change either early or late after recovery. The unique behavior of the case of Type I pneumonia, treated with specific anti-serum, whose blood abruptly lost resistance is a single exception. Even in this 


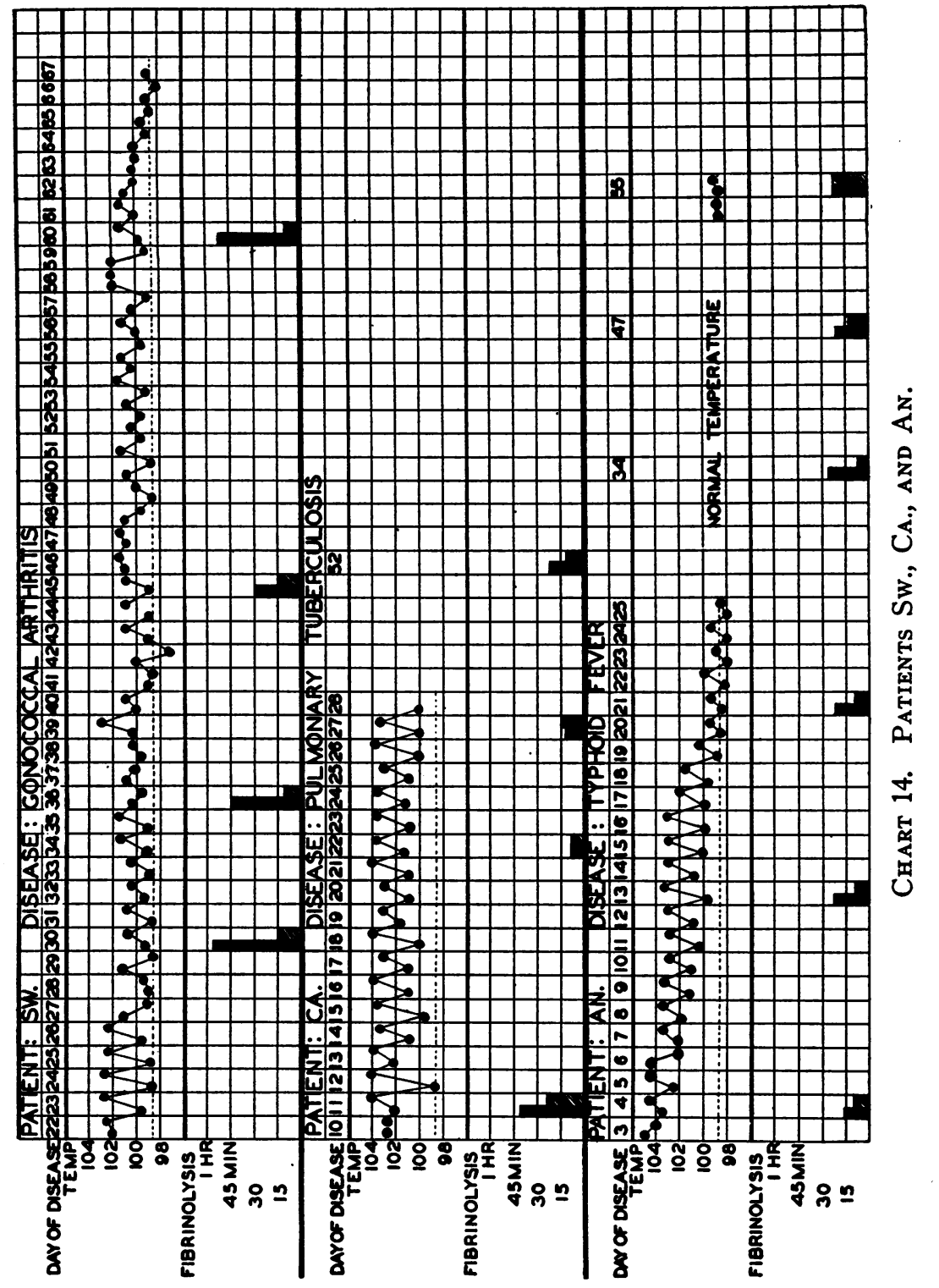




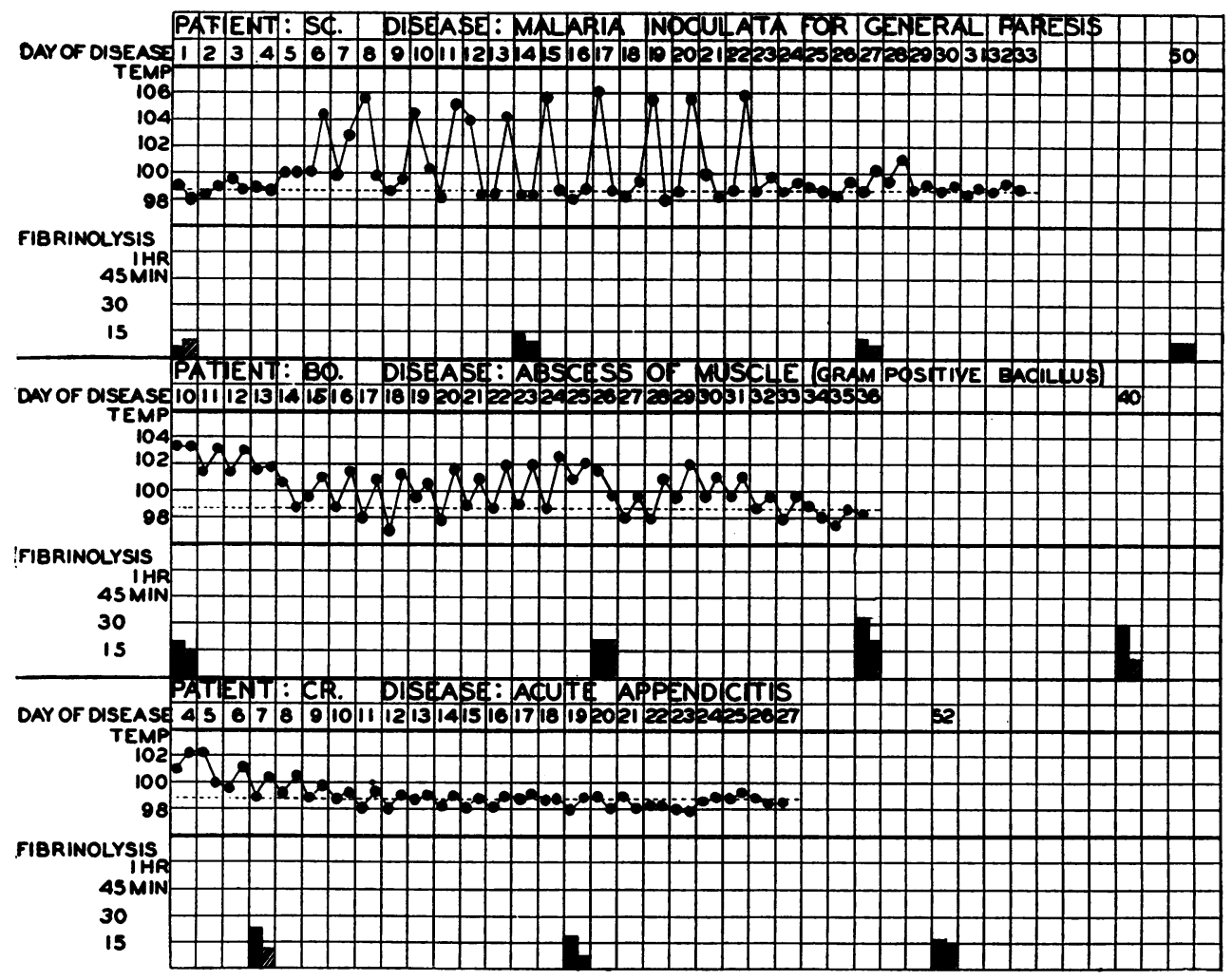

Chart 15. Patients Sc., Bo., and Cr.

case, the changes were the reverse of the course in patients with hemolytic streptococcus infections.

Repeated fibrinolytic tests with the plasma-clot from normal healthy adults

The observations recorded in Chart 16 were made with samples of blood obtained from normal adults, who were separately tested over a period of several months. The purpose of this study was to determine what variations in fibrinolytic rate occurred in an individual in whom the factor of disease was not present. Five normal persons were, therefore, bled at weekly or monthly intervals for 4 months. During this time they had no acute illnesses except mild coryza in the early spring.

The plasma-clot from the blood of individuals T., O., and G. were highly susceptible to fibrinolysis on every test. Among these three the greatest variation was with the blood of O., which on one test required 10 minutes for dissolution and on another required 40 minutes.

The blood of individuals $S$. and F. possessed, at the beginning, definite resistance to fibrinolysis, the plasma-clot of S.'s blood requiring 4 hours 
to liquefy, and that of F.'s requiring 3 hours. The activity of the test culture was proved by suitable control. The delayed rate of dissolution present in the fibrin from the initial bleeding remained essentially the same in each of 5 specimens of blood obtained at intervals for about 4 months.

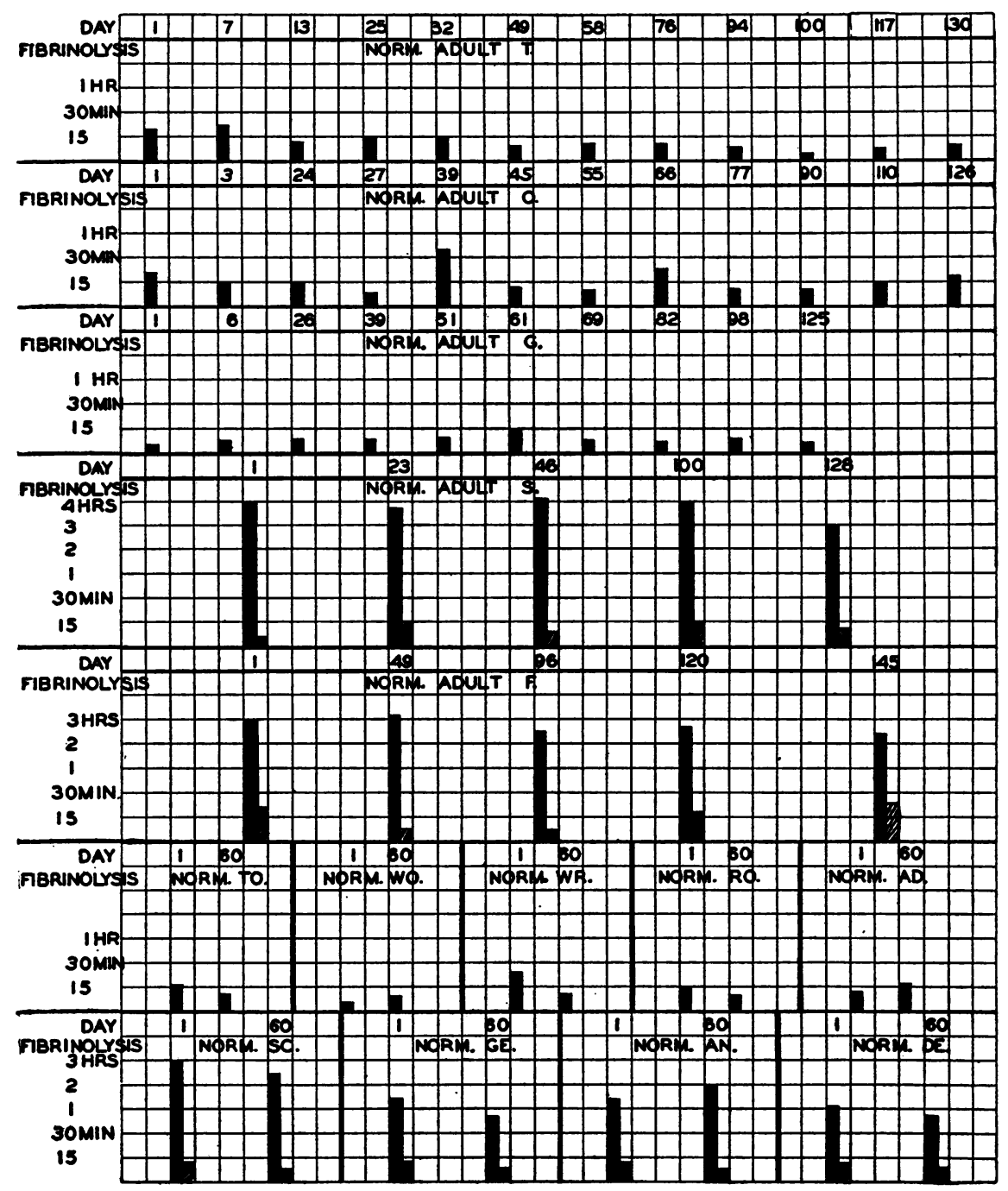

Chart 16. Normal Adults

Neither of these individuals gave a history of acute infections during the past few years.

In fibrinolytic tests with the blood of these five normal adults, therefore, it may be seen that, at least for as long as 4 months, the individual reactivity is not subject to significant variation. 
The uniformity of the individual reactivity is also evident in the tests which are recorded in the two bottom rows of Chart 16. These observations consist of 2 separate fibrinolytic tests with blood from each of 9 healthy adults. The samples of blood were taken 2 months apart. Individuals To., Wo., Wr., Ro. and Ad., were highly susceptible in each test, whereas the others, Sc., Ge., An. and De., possessesd definite resistance which was maintained with only slight variation.

The presence of some degree of anti-fibrinolytic resistance in the blood of a few healthy adults may be interpreted as an enduring partial response to some earlier infection or as an individual property natural to the person's blood. From all the charts it may be noted that there is frequently some discrepancy between the patient's blood and the normal control. Since it was impossible to know the behavior of the patient's blood before the disease began, the result of tests with the first bleeding cannot be accurately evaluated. It would seem, however, to be due either to the individuals own natural reactivity or to an immediate response to infection. One factor of infection, which undoubtedly contributes to the results, is the quantitative increase in blood fibrinogen which is known to occur early in many acute infections. The answer to this phase of the problem must await further study.

\section{DISCUSSION}

The observations, which comprise this report, are part of an investigation of the fibrinolytic properties of hemolytic streptococci. It has been of interest to attempt to determine whether or not this biological property of the organisms may be significant either as a factor in virulence or as an agent capable of evoking an immunological response. A study of the relationship of bacterial fibrinolysis to tissue invasion is now in progress but is not complete. The observations, which are given in detail in this article, concern the immunological phase of the problem.

The results, indicate that, following acute infection with hemolytic streptococci, the blood of the patient acquires a new property by means of which the plasma-clot becomes highly resistant to dissolution by active cultures of hemolytic streptococci.

The resistance, stimulated by acute streptococcus infection, was demonstrable in the blood of patients convalescent from erysipelas, scarlet fever, and acute tonsillitis. The time in the course of convalescence, at which maximum resistance to fibrinolysis was demonstrable, varied from an immediate response to a delay of several weeks. Some of the cases developed suppurative complications. Among these there was a tendency for resistance to appear more quickly than in the uncomplicated cases. This result suggests that the presence of pus may hasten the appearance of antifibrinolytic substances. It will be of interest, therefore, to investigate, in 
a larger series of cases, the relationship of suppuration to anti-fibrinolytic resistance, and to observe whether the character of the exudate changes in accordance with the ability to withstand the lytic power of the infecting agent.

In contrast to the anti-fibrinolytic response which follows streptococcus infection, the blood from patients with other acute illnesses did not contain significant amounts of anti-fibrinolytic substances effective against the active principle of streptococci, nor was convalescence in this control group of non-streptococcal infections attended by any change in rate of fibrinolysis.

Observations of clot dissolution with blood from healthy adults and from cases of low grade chronic disorders reveal, in about 75 per cent of the tests, high susceptibility to fibrinolysis. The varying degrees of resistance exhibited by the blood of the remaining 25 per cent cannot yet be interpreted. It seems probable either that it represents evidence of a previous streptococcus infection or indicates "natural immunity." Repeated tests on healthy adults show that, over a period of several months, the individual fibrinolytic rate does not significantly change; individuals, whose blood manifested susceptibility, remained so, and the plasma-clot of others, which was moderately resistant, maintained the same delayed rate of liquefaction. The constancy of the reaction in normal persons adds additional significance to the changes which occur following acute streptococcus disease.

The results, therefore, of repeated fibrinolytic tests with blood from cases of acute hemolytic streptococcus infections, and of comparable observations with blood from other types of acute infection, as well as from healthy individuals, have been interpreted as signifying that insusceptibility to fibrinolysis is specifically induced; that the fibrinolysin of hemolytic streptococci, in the body, evokes a definite response directed against the lytic action of the bacteria. The exact nature of anti-fibrinolytic substances, however, has not yet been determined.

Although the observations recorded in this article do not concern the fibrinolytic activity of hemolytic streptococci from the standpoint of bacterial pathogenicity, the fact that patients respond to infection by acquiring a means of combating the agent of fibrinolysis, might be considered as indirect evidence that this bacterial property is of some significance. It is, therefore, justifiable to comment briefly upon the possible rôle which a biological substance having the capacity to dissolve fibrin with great rapidity, might play in relation to bacterial virulence.

If the deposition of fibrin, which occurs early in the process of inflammation, is part of the attempt to wall off the infecting agent it becomes evident that the invasion of tissues might be influenced by bacterial fibrinolysin. Micro-organisms, under the circumstances, which are capable of breaking down the fibrin wall by dissolution would-barring other im- 
munological processes such as agglutination or phagocytosis-be free to spread through the tissues.

When the clinical course of certain acute fulminating hemolytic streptococcus infections is considered in the light of the highly active fibrinolytic properties of cultures of this organism, a causal relationship suggests itself. For example, the thin character of the exudative reactions, especially notable in acute streptococcus infections of serous surfaces, may find a rational interpretation on the basis of the liquefying property of these organisms. The walling-off process of recovery, which often consists of a barrier of fibrin deposition, may represent the anti-fibrinolytic immune reaction exemplified by the results contained in this article. The study of the relation of streptococcal fibrinolysis to virulence is proceeding along the lines indicated by the interpretation just mentioned.

\section{CONCLUSIONS}

Under the experimental conditions described, the plasma-clot from the blood of patients convalescent from acute hemolytic streptococcus infections was found to be highly resistant to fibrinolytic principles contained in active cultures of hemolytic streptococci. By repeated tests with samples of blood, obtained before and after recovery, it was possible to demonstrate, in the same individual, the development of anti-fibrinolysis.

In comparable tests with the blood from a limited number of other types of bacterial infection, the recovery of the patients was not followed by the appearance of anti-fibrinolytic substances in the blood.

The plasma-clot of the blood from 75 per cent of sixty so-called " normal" persons was highly susceptible to fibrinolysis.

\section{BIBLIOGRAPHY}

1. Tillett, W. S., and Garner, R. L., The fibrinolytic activity of hemolytic streptococci. J. Exper. Med., 1933, 58, 485. 